\title{
Chiral $\alpha$-Branched Benzylic Carbocations: Diastereoselective Intermolecular Reactions with Arene Nucleophiles and NMR- Spectroscopic Studies
}

\author{
Friedrich Mühlthau, ${ }^{\mathrm{a}}$ Daniel Stadler, ${ }^{\mathrm{a}}$ Alain Goeppert, ${ }^{\mathrm{b}}$ George A. Olah, ${ }^{\mathrm{b}}$ G. K. Surya Prakash, ${ }^{\mathrm{b}}$ \\ and Thorsten Bach $^{\mathrm{a}^{*}}$ \\ a Lehrstuhl für Organische Chemie I, Technische Universität München, \\ D-85747 Garching, Germany \\ thorsten.bach@ch.tum.de \\ ${ }^{\mathrm{b}}$ Loker Hydrocarbon Research Institute, University of Southern California, \\ Los Angeles, CA 90089-1062
}

\section{Supporting Information}

1. Preparation of the Starting Materials (+)-syn-1a, $(-)-\operatorname{syn}-\mathbf{1 a}, \mathbf{1 b}, \mathbf{5}$, and $\mathbf{6}$

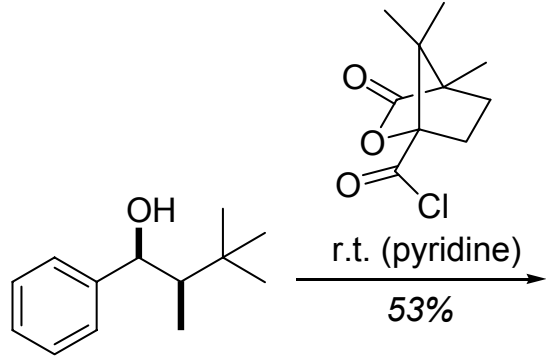

syn-1a

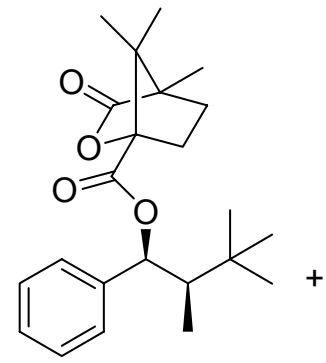

9<smiles>C[C@H]([C@H](OC(=O)C12CCC(C)(C(=O)O1)C2(C)C)c1ccccc1)C(C)(C)C</smiles>

10

syn-2,3,3-Trimethyl-1-phenyl-butan-1-ol (syn-1a, $875 \mathrm{mg}, 4.55 \mathrm{mmol}$ ) was placed in a flame dried flask and dissolved in dry pyridine $(15 \mathrm{~mL})$. After cooling the solution to $0{ }^{\circ} \mathrm{C},(-)$ camphanyl chloride $(1.28 \mathrm{~g}, 5.92 \mathrm{mmol})$ was added and the mixture was stirred for $48 \mathrm{~h}$ at room temperature. The reaction was stopped by adding water $(60 \mathrm{~mL})$ and the aqueous phase was extracted with dichloromethane $(3 \times 20 \mathrm{~mL})$. After rinsing the organic phase with water $(30 \mathrm{~mL})$ and sat. $\mathrm{NaCl}$ solution $(30 \mathrm{~mL})$ it was dried over $\mathrm{Na}_{2} \mathrm{SO}_{4}$, filtered and the solvent was evaporated. The originated diastereo isomers $\mathbf{9}$ and $\mathbf{1 0}$ were separated by column chromatography (pentane/Et $\left.t_{2} \mathrm{O} 90 / 10\right)$ yielding product $9(360 \mathrm{mg}, 0.97 \mathrm{mmol}, 21 \%)$ as a colorless oil in a diastereomeric ratio of $97 / 3$, product $10(354 \mathrm{mg}, 0.95 \mathrm{mmol}, 21 \%)$ as a white solid in a diastereomeric ratio of $98 / 2$ and a mixture of both $(192 \mathrm{mg}, 0.52 \mathrm{mmol}, 11 \%)$ as a colorless oil in a diastereomeric ratio of $60 / 40$.

4,7,7,-Trimethyl-3-oxo-2-oxa-bicyclo[2.2.1] heptane-1-carboxylic acid (1S,2R)-2,3,3-trimethyl-1phenyl-butyl ester (9)

$$
\begin{aligned}
& \boldsymbol{R}_{\mathbf{f}} 0.13\left(\mathrm{P} / \mathrm{Et}_{2} \mathrm{O}=90 / 10\right) \\
& {[\boldsymbol{\alpha}]_{\mathbf{D}}{ }^{\mathbf{2 0}}+8.2\left(c=1.04, \mathrm{CH}_{2} \mathrm{Cl}_{2}\right)}
\end{aligned}
$$


IR (film): $\tilde{v} 3062 \mathrm{~cm}^{-1}$ (w), 2968 (s), 1790 (vs), 1732 (vs), 1269 (s), 1168 (m), 1100 (s), 1059 (m), $738(\mathrm{~m})$.

${ }^{1}$ H-NMR $\left(\mathrm{CDCl}_{3}, 360 \mathrm{MHz}, 297 \mathrm{~K}\right): \delta 0.95(\mathrm{~s}, 3 \mathrm{H}), 0.97\left(\mathrm{~d},{ }^{3} J=7.1 \mathrm{~Hz}, 3 \mathrm{H}\right), 0.99(\mathrm{~s}, 9 \mathrm{H})$, $1.06(\mathrm{~s}, 3 \mathrm{H}), 1.12$ (s, $3 \mathrm{H}), 1.59\left(\mathrm{dq},{ }^{3} J=1.7 \mathrm{~Hz},{ }^{3} J=7.1 \mathrm{~Hz}, 1 \mathrm{H}\right), 1.70$ (ddd, ${ }^{2} J=13.2 \mathrm{~Hz},{ }^{3} J=$ $\left.4.2 \mathrm{~Hz},{ }^{3} J=9.3 \mathrm{~Hz}, 1 \mathrm{H}\right), 1.92\left(\mathrm{ddd},{ }^{2} J=13.2 \mathrm{~Hz},{ }^{3} J=4.6 \mathrm{~Hz},{ }^{3} J=10.7 \mathrm{~Hz}, 1 \mathrm{H}\right.$ ), 2.05 (ddd, ${ }^{2} J=$ $\left.13.4 \mathrm{~Hz},{ }^{3} J=4.6 \mathrm{~Hz},{ }^{3} J=9.3 \mathrm{~Hz}, 1 \mathrm{H}\right), 2.40\left(\mathrm{ddd},{ }^{2} J=13.4 \mathrm{~Hz},{ }^{3} J=4.2 \mathrm{~Hz},{ }^{3} J=10.7 \mathrm{~Hz}, 1 \mathrm{H}\right)$, 6.28 (bs, $1 \mathrm{H}), 7.22-7.33$ (m, $5 \mathrm{H})$.

${ }^{13}$ C-NMR $\left(\mathrm{CDCl}_{3}, 90.6 \mathrm{MHz}, 297 \mathrm{~K}\right): \delta 7.9$ (q), 9.6 (q), 16.7 (q), 16.7 (q), 28.2 (q), 28.9 (t), 31.0 (t), $33.4(\mathrm{~s}), 49.7$ (d), 54.2 (s), 54.8 (s), 76.4 (d), 91.1 (s), 125.5 (d), 127.2 (d), 128.2 (d), 141.6 (s), $167.1(\mathrm{~s}), 178.5(\mathrm{~s})$.

MS (EI, $70 \mathrm{eV}): m / z$ (\%) 372 (2), 287 (38), 182 (100), 164 (50), 153 (17), 136 (48), 125 (72), 118 (34), 105 (15), 97 (26), 83 (43), 57 (34).

HRMS Calcd. for $\mathrm{C}_{23} \mathrm{H}_{32} \mathrm{O}_{4}$ (372.50): 372.2301; found: 372.2291 .

4,7,7,-Trimethyl-3-oxo-2-oxa-bicyclo[2.2.1] heptane-1-carboxylic acid (1R,2S)-2,3,3-trimethyl-1phenyl-butyl ester (10)

$\boldsymbol{R}_{\mathbf{f}} 0.07\left(\mathrm{P} / \mathrm{Et}_{2} \mathrm{O}=90 / 10\right)$

$[\boldsymbol{\alpha}]_{\mathbf{D}}{ }^{\mathbf{2 0}}-18.9\left(c=1.01, \mathrm{CH}_{2} \mathrm{Cl}_{2}\right)$

IR (KBr): $\tilde{v} 2971 \mathrm{~cm}^{-1}$ (s), 1787 (vs), 1732 (vs), 1270 (s), 1172 (m), 1102 (s), 1060 (m), 786 (s).

${ }^{1}$ H-NMR $\left(\mathrm{CDCl}_{3}, 360 \mathrm{MHz}, 297 \mathrm{~K}\right): \delta 0.96(\mathrm{~s}, 3 \mathrm{H}), 0.98\left(\mathrm{~d},{ }^{3} J=7.1 \mathrm{~Hz}, 3 \mathrm{H}\right), 0.99(\mathrm{~s}, 9 \mathrm{H})$, $1.11(\mathrm{~s}, 3 \mathrm{H}), 1.12(\mathrm{~s}, 3 \mathrm{H}), 1.61\left(\mathrm{dq},{ }^{3} J=1.6 \mathrm{~Hz},{ }^{3} J=7.1 \mathrm{~Hz}, 1 \mathrm{H}\right), 1.70\left(\mathrm{ddd},{ }^{2} J=13.2 \mathrm{~Hz},{ }^{3} J=\right.$ $\left.4.3 \mathrm{~Hz},{ }^{3} J=9.3 \mathrm{~Hz}, 1 \mathrm{H}\right), 1.92\left(\mathrm{ddd},{ }^{2} J=13.2 \mathrm{~Hz},{ }^{3} J=4.6 \mathrm{~Hz},{ }^{3} J=10.7 \mathrm{~Hz}, 1 \mathrm{H}\right), 2.06\left(\mathrm{ddd},{ }^{2} J=\right.$ $\left.13.4 \mathrm{~Hz},{ }^{3} J=4.6 \mathrm{~Hz},{ }^{3} J=9.3 \mathrm{~Hz}, 1 \mathrm{H}\right), 2.39\left(\mathrm{ddd},{ }^{2} J=13.4 \mathrm{~Hz},{ }^{3} J=4.3 \mathrm{~Hz},{ }^{3} J=10.7 \mathrm{~Hz}, 1 \mathrm{H}\right)$, $6.23(\mathrm{bs}, 1 \mathrm{H}), 7.22-7.33(\mathrm{~m}, 5 \mathrm{H})$.

${ }^{13} \mathbf{C}-\mathbf{N M R}\left(\mathrm{CDCl}_{3}, 90.6 \mathrm{MHz}, 297 \mathrm{~K}\right): \delta 8.1$ (q), 9.6 (q), 16.8 (q), 16.9 (q), $28.2(\mathrm{q}), 29.0$ (t), 31.2 (t), 33.4 (s), 49.4 (d), 54.1 (s), 54.9 (s), 76.7 (d), 91.2 (s), 125.4 (d), 127.3 (d), 128.3 (d), 141.7 (s), $167.0(\mathrm{~s}), 178.3(\mathrm{~s})$.

MS (EI, $70 \mathrm{eV}): m / z$ (\%) 372 (2), 287 (35), 182 (100), 164 (49), 153 (15), 136 (46), 125 (69), 118 (37), 105 (15), 97 (25), 83 (42), 57 (35).

Anal. Calcd. for $\mathrm{C}_{23} \mathrm{H}_{32} \mathrm{O}_{4}$ (372.50): C, 74.16; H, 8.66; found: C, 74.11; H, 8.86.

Synthesis of (+)-syn-2,3,3-Trimethyl-1-phenyl-butan-1-ol [(+)-syn-1a]

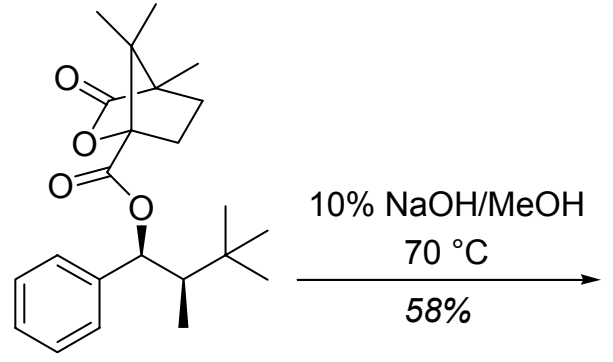

9<smiles>C[C@H]([C@@H](O)c1ccccc1)C(C)(C)C</smiles>

(+)-syn-1a 
Substrate 9 (342 $\mathrm{mg}, 0.92 \mathrm{mmol})$ was dissolved in methanol $(20 \mathrm{~mL})$ and $10 \%$ aqueous solution of $\mathrm{NaOH}(5 \mathrm{~mL})$ and refluxed for two hours. Water was added $(50 \mathrm{~mL})$ and the aqueous phase was extracted with diethylether $(3 \times 20 \mathrm{~mL})$. After rinsing the organic phase with sat. $\mathrm{NaCl}$ solution $(30 \mathrm{~mL})$ it was dried over $\mathrm{Na}_{2} \mathrm{SO}_{4}$, filtered and the solvent was evaporated. Column chromatography (pentane/Et $t_{2} \mathrm{O}$ 90/10) yielded the product (+)-syn-1a (103 mg, $\left.0.54 \mathrm{mmol}, 58 \%\right)$ as a colorless oil.

$\boldsymbol{R}_{\mathbf{f}} 0.23\left(\mathrm{P} / \mathrm{Et}_{2} \mathrm{O}=90 / 10\right)$

$[\alpha]_{\mathbf{D}}{ }^{\mathbf{2 0}}+45.7\left(c=1.13, \mathrm{CH}_{2} \mathrm{Cl}_{2}\right)$

${ }^{1}$ H-NMR $\left(\mathrm{CDCl}_{3}, 360 \mathrm{MHz}, 297 \mathrm{~K}\right): \delta 0.79\left(\mathrm{~d},{ }^{3} J=7.1 \mathrm{~Hz}, 3 \mathrm{H}\right), 1.06(\mathrm{~s}, 9 \mathrm{H}), 1.51$ (dq, ${ }^{3} J=$ $\left.1.5 \mathrm{~Hz},{ }^{3} J=7.1 \mathrm{~Hz}, 1 \mathrm{H}\right), 1.56$ (bs, $\left.1 \mathrm{H}\right), 5.16$ (bs, $\left.1 \mathrm{H}\right), 7.20-7.25(\mathrm{~m}, 1 \mathrm{H}), 7.30-7.36(\mathrm{~m}, 4 \mathrm{H})$.

${ }^{13} \mathbf{C}-\mathbf{N M R}\left(\mathrm{CDCl}_{3}, 90 \mathrm{MHz}, 297 \mathrm{~K}\right): \delta 6.6$ (q), 28.5 (q), 33.4 (s), 50.0 (d), 73.2 (d), 125.4 (d), 126.5 (d), 128.0 (d), 146.1 (s).

MS (EI, $70 \mathrm{eV}$ ): m/z (\%) 192 (3), 117 (3), 107 (100), 85 (5), 79 (23), 77 (12), 57 (5).

Synthesis of (-)-syn-2,3,3-Trimethyl-1-phenyl-butan-1-ol [(-)-syn-1a]

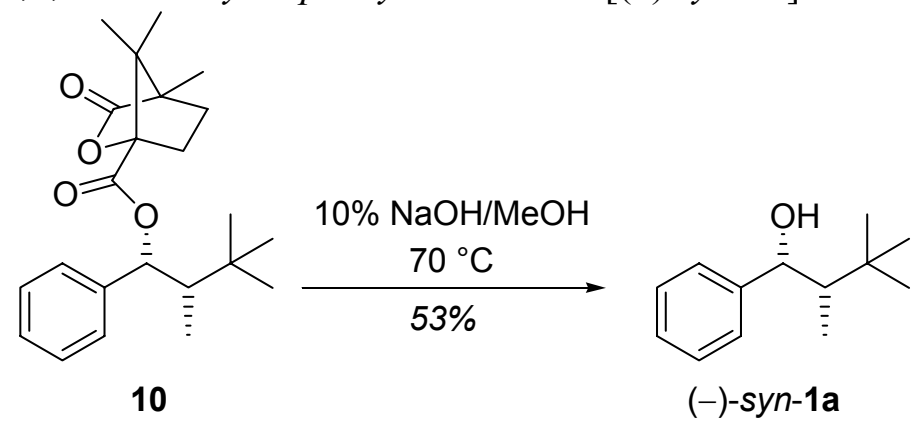

Substrate 10 (284 mg, $0.76 \mathrm{mmol})$ was dissolved in methanol $(20 \mathrm{~mL})$ and $10 \%$ aqueous solution of $\mathrm{NaOH}(5 \mathrm{~mL})$ and refluxed for two hours. Water was added $(50 \mathrm{~mL})$ and the aqueous phase was extracted with diethylether $(3 \times 20 \mathrm{~mL})$. After rinsing the organic phase with sat. $\mathrm{NaCl}$ solution $(30 \mathrm{~mL})$ it was dried over $\mathrm{Na}_{2} \mathrm{SO}_{4}$, filtered and the solvent was evaporated. Column chromatography (pentane/Et $\mathrm{t}_{2} \mathrm{O}$ 90/10) yielded the product (-)-syn-1a (78 mg, $\left.0.41 \mathrm{mmol}, 53 \%\right)$ as a colorless oil.

$\boldsymbol{R}_{\mathbf{f}} 0.23\left(\mathrm{P} / \mathrm{Et}_{2} \mathrm{O}=90 / 10\right)$

$[\boldsymbol{\alpha}]_{\mathbf{D}}{ }^{\mathbf{2 0}}-45.1\left(c=1.05, \mathrm{CH}_{2} \mathrm{Cl}_{2}\right)$

${ }^{1}$ H-NMR $\left(\mathrm{CDCl}_{3}, 360 \mathrm{MHz}, 297 \mathrm{~K}\right): \delta 0.79\left(\mathrm{~d},{ }^{3} J=7.1 \mathrm{~Hz}, 3 \mathrm{H}\right), 1.06(\mathrm{~s}, 9 \mathrm{H}), 1.51$ (dq, ${ }^{3} J=$ $\left.1.5 \mathrm{~Hz},{ }^{3} J=7.1 \mathrm{~Hz}, 1 \mathrm{H}\right), 1.56$ (bs, $\left.1 \mathrm{H}\right), 5.16$ (bs, $\left.1 \mathrm{H}\right), 7.20-7.25(\mathrm{~m}, 1 \mathrm{H}), 7.30-7.36(\mathrm{~m}, 4 \mathrm{H})$.

${ }^{13} \mathbf{C}-\mathbf{N M R}\left(\mathrm{CDCl}_{3}, 90 \mathrm{MHz}, 297 \mathrm{~K}\right): \delta 6.6$ (q), 28.5 (q), 33.4 (s), 50.0 (d), 73.2 (d), 125.4 (d), 126.5 (d), 128.0 (d), 146.1 (s).

MS (EI, $70 \mathrm{eV}): m / z$ (\%) 192 (3), 117 (3), 107 (100), 85 (5), 79 (23), 77 (12), 57 (5). 
Synthesis of anti-3,4,4-Trimethyl-2-phenyl-pentan-2-ol (1b)

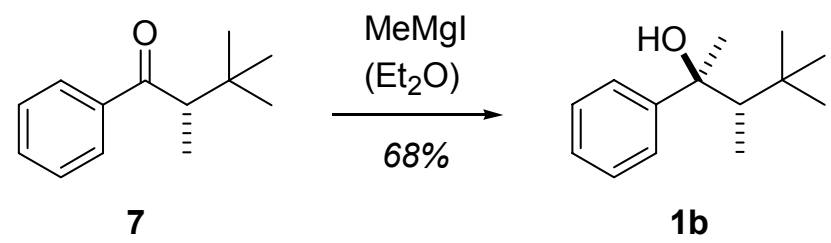

Magnesium (0.49 g, $20.0 \mathrm{mmol}$ ) was flame dried under vacuum in a flask with a reflux condenser. In an argon atmosphere diethylether $(5 \mathrm{~mL})$ and one fifth of methyl iodide $(2.13 \mathrm{~g}, 15$ mmol) was added. After gentle warming the formation of the Grignard reagent started and was kept alive through slow addition of the residual methyl iodide. The solution was diluted with diethylether $(15 \mathrm{~mL})$ and stirred for one hour at room temperature. 1-Phenyl-2,3,3-trimethylbutan-1-on $(7,1.90 \mathrm{~g}, 10.0 \mathrm{mmol})$ was placed in a flame dried flask and dissolved in dry diethylether $(20 \mathrm{~mL})$. After cooling the solution to $0{ }^{\circ} \mathrm{C}$, the above mentioned Grignard solution was added and the mixture was stirred for two hours at room temperature. The reaction was stopped by adding sat. $\mathrm{NH}_{4} \mathrm{Cl}$ solution $(25 \mathrm{~mL})$ and the aqueous phase was extracted with diethylether $(25 \mathrm{~mL})$. After rinsing the organic phase with water $(25 \mathrm{~mL})$ and sat. $\mathrm{NaCl}$ solution $(25 \mathrm{~mL})$ it was dried over $\mathrm{Na}_{2} \mathrm{SO}_{4}$, filtered and the solvent was evaporated. Column chromatography (pentane/ $\mathrm{Et}_{2} \mathrm{O}$ 90/10) yielded the product $\mathbf{1 b}(1.40 \mathrm{~g}, 6.79 \mathrm{mmol}, 68 \%)$ as a colorless oil in diastereomerical pure form.

$\boldsymbol{R}_{\mathbf{f}} 0.30\left(\mathrm{P} / \mathrm{Et}_{2} \mathrm{O}=90 / 10\right)$

IR (film): $\tilde{v} 3488 \mathrm{~cm}^{-1}$ (w), 3086 (w), 2957 (s), 1493 (m), 1446 (m), 1029 (m), 766 (s), 703 (s).

${ }^{1}$ H-NMR $\left(\mathrm{CDCl}_{3}, 360 \mathrm{MHz}, 297 \mathrm{~K}\right): \delta 0.89\left(\mathrm{~d},{ }^{3} \mathrm{~J}=7.2 \mathrm{~Hz}, 3 \mathrm{H}\right), 0.91(\mathrm{~s}, 9 \mathrm{H}), 1.54(\mathrm{~s}, 3 \mathrm{H})$, $1.66(\mathrm{~s}, 1 \mathrm{H}), 1.92\left(\mathrm{q},{ }^{3} J=7.2 \mathrm{~Hz}, 1 \mathrm{H}\right), 7.17-7.24(\mathrm{~m}, 1 \mathrm{H}), 7.27-7.35(\mathrm{~m}, 2 \mathrm{H}), 7.42-7.49(\mathrm{~m}$, $2 \mathrm{H})$.

${ }^{13}$ C-NMR $\left(\mathrm{CDCl}_{3}, 90.6 \mathrm{MHz}, 297 \mathrm{~K}\right): \delta 13.2$ (q), 29.5 (q), 30.5 (q), 35.2 (s), 50.9 (d), 79.0 (s), 125.2 (d), 126.3 (d), $128.0(\mathrm{~d}), 151.4(\mathrm{~s})$.

MS (EI, $70 \mathrm{eV}): m / z(\%) 206(<1), 132$ (17), 122 (34), 121 (100), 105 (18), 57 (24), 43 (95).

Anal. Calcd. for $\mathrm{C}_{14} \mathrm{H}_{22} \mathrm{O}$ (206.32): C, 81.50; H, 10.75; found: C, 81.26; H, 10.82 .

Synthesis of syn-1-(4-Chloro-phenyl)-2,3,3-trimethyl-butan-1-ol (syn-5)<smiles>C[C@H](C=O)C(C)(C)C</smiles>

8<smiles>CCOc1ccc(N(C)c2ccc(Cl)cc2)cc1</smiles><smiles>C[C@H]([C@@H](O)c1ccc(Cl)cc1)C(C)(C)C</smiles>

5

Magnesium (321 mg, $13.2 \mathrm{mmol}$ ) was flame dried under vacuum in a flask with a reflux condenser. In an argon atmosphere diethylether $(1.2 \mathrm{~mL})$ and one fifth of 4-bromochlorobenzene $(1.26 \mathrm{~g}, 6.58 \mathrm{mmol})$ was added. After gentle warming the formation of the Grignard reagent started and was kept alive through slow addition of the residual 4-bromochlorobenzene. The 
solution was diluted with diethylether $(4.8 \mathrm{~mL})$ and stirred for one hour at room temperature. 2,3,3-Trimethylbutanal $(\mathbf{8}, 1.90 \mathrm{~g}, 5.00 \mathrm{mmol})$ was placed in a flame dried flask and dissolved in dry diethylether $(20 \mathrm{~mL})$. After cooling the solution to $0{ }^{\circ} \mathrm{C}$, the above mentioned Grignard solution was added and the mixture was stirred over night at room temperature. The reaction was stopped by adding sat. $\mathrm{NH}_{4} \mathrm{Cl}$ solution $(20 \mathrm{~mL})$ and the aqueous phase was extracted with diethylether $(20 \mathrm{~mL})$. After rinsing the organic phase with water $(2 \times 20 \mathrm{~mL})$ and sat. $\mathrm{NaCl}$ solution $(20 \mathrm{~mL})$ it was dried over $\mathrm{Na}_{2} \mathrm{SO}_{4}$, filtered and the solvent was evaporated. Column chromatography (pentane/ $\mathrm{Et}_{2} \mathrm{O}$ 90/10) yielded the product $5(588 \mathrm{mg}, 2.59 \mathrm{mmol}, 52 \%)$ as a colorless oil in a diastereomeric ratio of syn/anti $98 / 2$.

$\boldsymbol{R}_{\mathbf{f}} 0.25\left(\mathrm{P} / \mathrm{Et}_{2} \mathrm{O}=90 / 10\right)$

mp. $75^{\circ} \mathrm{C}$

IR (KBr): ̃ $3386 \mathrm{~cm}^{-1}$ (m), 2957 (m), 1491 (vs), 1400 (m), 1364 (m), 1090 (s), 1056 (m), 1012 (s), 973 (s), 849 (s), 806 (vs), 669 (m), 532 (m).

${ }^{1}$ H-NMR $\left(\mathrm{CDCl}_{3}, 360 \mathrm{MHz}, 297 \mathrm{~K}\right): \delta 0.76\left(\mathrm{~d},{ }^{3} J=7.1 \mathrm{~Hz}, 3 \mathrm{H}\right), 1.05(\mathrm{~s}, 9 \mathrm{H}), 1.45$ (qd, ${ }^{3} J=$ $\left.7.1 \mathrm{~Hz},{ }^{3} J=1.5 \mathrm{~Hz}, 1 \mathrm{H}\right), 1.56$ (bs, $\left.1 \mathrm{H}\right), 5.13$ (bs, $\left.1 \mathrm{H}\right), 7.22-7.33(\mathrm{~m}, 4 \mathrm{H})$.

${ }^{13} \mathbf{C}-\mathbf{N M R}\left(\mathrm{CDCl}_{3}, 90.6 \mathrm{MHz}, 297 \mathrm{~K}\right): \delta 6.7$ (q), 28.6 (q), 33.6 (s), 50.2 (d), 72.8 (d), 126.9 (d), 128.3 (d), 132.3 (s), 144.6 (s).

MS (EI, $70 \mathrm{eV}): m / z$ (\%) 226 (2), 143 (29), 141 (100), 113 (21), 77 (63), 57 (26) 55 (26).

Anal. Calcd. for $\mathrm{C}_{13} \mathrm{H}_{19} \mathrm{ClO}$ (226.74): C, 68.86; H, 8.45; Cl, 15.43; found: $\mathrm{C}, 68.77 ; \mathrm{H}, 8.48 ; \mathrm{Cl}$, 15.18 .

Synthesis of syn-4-(1-Hydroxy-2,3,3-trimethyl-butyl)-benzonitril (syn-6)

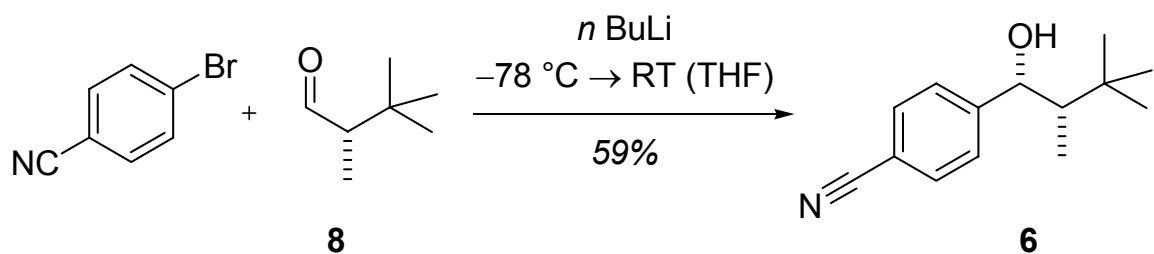

4-Bromobenzonitril $(910 \mathrm{mg}, 5.00 \mathrm{mmol})$ was dissolved in dry tetrahydrofuran $(30 \mathrm{~mL})$ and cooled to $-78{ }^{\circ} \mathrm{C}$. After the addition of $n$-butyl lithium $(2.00 \mathrm{~mL}$ of a $2.5 \mathrm{M}$ solution in hexane, $5.00 \mathrm{mmol})$ the solution was stirred for 30 minutes. 2,3,3-Trimethylbutanal $(\mathbf{8}, 570 \mathrm{mg}, 5.00$ $\mathrm{mmol}$ ) was added and the reaction mixture was warmed to room temperature over one hour. The reaction was stopped by adding sat. $\mathrm{NH}_{4} \mathrm{Cl}$ solution $(30 \mathrm{~mL})$ and the aqueous phase was extracted with diethylether $(30 \mathrm{~mL})$. After rinsing the organic phase with water $(30 \mathrm{~mL})$ and sat. $\mathrm{NaCl}$ solution $(30 \mathrm{~mL})$ it was dried over $\mathrm{Na}_{2} \mathrm{SO}_{4}$, filtered and the solvent was evaporated. Column chromatography (pentane/ $\left.\mathrm{Et}_{2} \mathrm{O} 90 / 10\right)$ yielded the product $6(638 \mathrm{mg}, 2.94 \mathrm{mmol}, 59 \%)$ as yellowish crystals in a diastereomeric ratio of syn/anti 96/4.

$\boldsymbol{R}_{\mathbf{f}} 0.21\left(\mathrm{P} / \mathrm{Et}_{2} \mathrm{O}=70 / 30\right)$

mp. $109^{\circ} \mathrm{C}$ 
IR (KBr): $\tilde{v} 3534 \mathrm{~cm}^{-1}$ (m), 2960 (m), 2227 (vs), 1605 (s), 1499 (m), 1364 (w), 1236 (m), 1102 (w), 1055 (m), $1016(\mathrm{~m}), 978(\mathrm{w}), 863(\mathrm{~m}), 842$ (w), $810(\mathrm{~m}), 765(\mathrm{w}), 662(\mathrm{w}), 560(\mathrm{vs})$.

${ }^{1}$ H-NMR $\left(\mathrm{CDCl}_{3}, 360 \mathrm{MHz}, 297 \mathrm{~K}\right): \delta 0.74\left(\mathrm{~d},{ }^{3} J=7.2 \mathrm{~Hz}, 3 \mathrm{H}\right), 1.06(\mathrm{~s}, 9 \mathrm{H}), 1.48$ (qd, ${ }^{3} J=$ $\left.7.2 \mathrm{~Hz},{ }^{3} J=1.4 \mathrm{~Hz}, 1 \mathrm{H}\right), 1.66\left(\mathrm{~d},{ }^{3} J=4.4 \mathrm{~Hz}, 1 \mathrm{H}\right), 5.20\left(\mathrm{~d},{ }^{3} J=4.4 \mathrm{~Hz}, 1 \mathrm{H}\right), 7.43\left(\mathrm{~d},{ }^{3} J=\right.$ $8.3 \mathrm{~Hz}, 2 \mathrm{H}), 7.62\left(\mathrm{~d},{ }^{3} J=8.3 \mathrm{~Hz}, 2 \mathrm{H}\right)$.

${ }^{13}$ C-NMR $\left(\mathrm{CDCl}_{3}, 90.6 \mathrm{MHz}, 297 \mathrm{~K}\right): \delta 6.7$ (q), 28.6 (q), 33.6 (s), 50.2 (d), 72.9 (d), 110.5 (s), $119.1(\mathrm{~s}), 126.4$ (d), $132.0(\mathrm{~d}), 151.6(\mathrm{~s})$.

MS (EI, $70 \mathrm{eV}): m / z(\%) 217$ (<1), 133 (100), 132 (28), 104 (22), 85 (22).

Anal. Calcd. for $\mathrm{C}_{14} \mathrm{H}_{19} \mathrm{NO}$ (217.31): C, 77.38; H, 77.38; N, 6.45; found: C, 77.12; H, 8.71; N, 6.41 . 
2. $\quad{ }^{1} \mathrm{H}$ - and ${ }^{13} \mathrm{C}-\mathrm{NMR}$ data for the substrates syn-1a, anti-1a, $\mathbf{2 a}, \mathbf{2 b}, \mathbf{3}$, and $\mathbf{4}$ Spectroscopic Data of syn-2,3,3-Trimethyl-1-phenyl-butan-1-ol (syn-1a)

${ }^{1}$ H-NMR $\left(\mathrm{CDCl}_{3}, 360 \mathrm{MHz}, 297 \mathrm{~K}\right): \delta 0.79\left(\mathrm{~d},{ }^{3} J=7.2 \mathrm{~Hz}, 3 \mathrm{H}\right), 1.06(\mathrm{~s}, 9 \mathrm{H}), 1.51$ (qd, ${ }^{3} J=$ $\left.7.2 \mathrm{~Hz},{ }^{3} J=1.5 \mathrm{~Hz}, 1 \mathrm{H}\right), 1.73$ (bs, $\left.1 \mathrm{H}\right), 5.16$ (bs, $\left.1 \mathrm{H}\right), 7.19-7.27$ (m, $\left.1 \mathrm{H}\right), 7.29-7.37$ (m, $\left.4 \mathrm{H}\right)$.

${ }^{13} \mathbf{C}-\mathrm{NMR}\left(\mathrm{CDCl}_{3}, 90 \mathrm{MHz}, 297 \mathrm{~K}\right): \delta 6.7$ (q), 28.6 (q), 33.6 (s), 50.2 (d), 73.3 (d), 125.5 (d), 126.7 (d), 128.2 (d), 146.2 (s).

Spectroscopic Data of anti-2,3,3-Trimethyl-1-phenyl-butan-1-ol (anti-1a)

${ }^{1}$ H NMR $\left(\mathrm{CDCl}_{3}, 360 \mathrm{MHz}, 297 \mathrm{~K}\right): \delta 0.61\left(\mathrm{~d},{ }^{3} J=7.0 \mathrm{~Hz}, 3 \mathrm{H}\right), 1.01(\mathrm{~s}, 9 \mathrm{H}), 1.65$ (bs, $\left.1 \mathrm{H}\right)$, $1.74\left(\operatorname{virt} \mathrm{p},{ }^{3} \mathrm{~J} \cong 7.3 \mathrm{~Hz}, 1 \mathrm{H}\right), 4.63\left(\mathrm{~d},{ }^{3} J=7.7 \mathrm{~Hz}, 1 \mathrm{H}\right), 7.23-7.36(\mathrm{~m}, 5 \mathrm{H})$.

${ }^{13} \mathbf{C ~ N M R}\left(\mathrm{CDCl}_{3}, 90 \mathrm{MHz}, 297 \mathrm{~K}\right): \delta 13.1$ (q), 28.9 (q), 33.4 (s), 49.0 (d), 77.6 (d), 127.4 (d), 127.6 (d), 128.4 (d), 145.2 (s).

Spectroscopic Data of 2-Methyl-1-phenyl-butan-1-ol (2a)

${ }^{1}$ H-NMR $\left(\mathrm{CDCl}_{3}, 360 \mathrm{MHz}, 297 \mathrm{~K}\right): \delta 0.75\left(\mathrm{~d},{ }^{3} J=6.8 \mathrm{~Hz}, 1.5 \mathrm{H}\right), 0.89\left(\mathrm{t},{ }^{3} J=7.6 \mathrm{~Hz}, 1.5 \mathrm{H}\right)$, $0.94\left(\mathrm{~d},{ }^{3} J=6.6 \mathrm{~Hz}, 1.5 \mathrm{H}\right), 0.94\left(\mathrm{t},{ }^{3} J=7.6 \mathrm{~Hz}, 1.5 \mathrm{H}\right), 1.03-1.28(\mathrm{~m}, 1 \mathrm{H}), 1.34-1.48(\mathrm{~m}, 0.5 \mathrm{H})$, $1.63-1.84(\mathrm{~m}, 2.5 \mathrm{H}), 4.44\left(\mathrm{~d},{ }^{3} J=7.0 \mathrm{~Hz}, 0.5 \mathrm{H}\right), 4.53\left(\mathrm{~d},{ }^{3} J=5.9 \mathrm{~Hz}, 0.5 \mathrm{H}\right), 7.23-7.39(\mathrm{~m}$, $5 \mathrm{H})$.

diastereoisomer A

${ }^{13}$ C-NMR $\left(\mathrm{CDCl}_{3}, 90 \mathrm{MHz}, 297 \mathrm{~K}\right): \delta 11.8$ (q), 14.1 (q), 26.0 (t), 42.1 (d), 79.0 (d), 126.5 (d), 127.4 (d), 128.3 (d), 144.1 (s).

diastereoisomer $\mathrm{B}$

${ }^{13}$ C-NMR $\left(\mathrm{CDCl}_{3}, 90 \mathrm{MHz}, 297 \mathrm{~K}\right): \delta 11.4$ (q), 15.3 (q), 25.0 (t), 41.8 (d), 78.2 (d), 126.9 (d), 127.6 (d), 128.3 (d), 143.8 (s).

Spectroscopic Data of 3-Methyl-2-phenyl-pentan-2-ol (2b)

${ }^{1}$ H-NMR $\left(\mathrm{CDCl}_{3}, 360 \mathrm{MHz}, 297 \mathrm{~K}\right): \delta 0.81-1.03(\mathrm{~m}, 7 \mathrm{H}), 1.45-1.77(\mathrm{~m}, 6 \mathrm{H}), 7.23\left(\mathrm{t},{ }^{3} \mathrm{~J}=\right.$ $7.3 \mathrm{~Hz}, 1 \mathrm{H}), 7.33$ (virt t, $\left.{ }^{3} J \cong 7.8 \mathrm{~Hz}, 2 \mathrm{H}\right), 7.42\left(\mathrm{~d},{ }^{3} J=7.5 \mathrm{~Hz}, 2 \mathrm{H}\right.$ ).

major diastereoisomer

${ }^{13}$ C-NMR $\left(\mathrm{CDCl}_{3}, 90 \mathrm{MHz}, 297 \mathrm{~K}\right): \delta 12.7$ (q), 13.7 (q), 24.0 (t), 26.5 (q), 46.0 (d), 77.2 (s), 125.5 (d), 126.6 (d), 128.0 (d), 148.1 (s).

minor diastereoisomer

${ }^{13}$ C-NMR $\left(\mathrm{CDCl}_{3}, 90 \mathrm{MHz}, 297 \mathrm{~K}\right): \delta 12.8$ (q), 13.5 (q), 24.1 (t), 26.8 (q), 46.0 (d), 77.4 (s), 125.4 (d), 126.5 (d), 128.0 (d), 148.4 (s). 
Spectroscopic Data of syn-1-(4-Methoxy-phenyl)-2,3,3-trimethyl-butan-1-ol (syn-3)

${ }^{1}$ H-NMR $\left(\mathrm{CDCl}_{3}, 360 \mathrm{MHz}, 297 \mathrm{~K}\right): \delta 0.79\left(\mathrm{~d},{ }^{3} J=7.2 \mathrm{~Hz}, 3 \mathrm{H}\right), 1.04(\mathrm{~s}, 9 \mathrm{H}), 1.76\left(\mathrm{qd},{ }^{3} J=\right.$ $\left.7.2 \mathrm{~Hz},{ }^{3} J=1.6 \mathrm{~Hz}, 1 \mathrm{H}\right), 1.51\left(\mathrm{~d},{ }^{3} J=3.9 \mathrm{~Hz}, 1 \mathrm{H}\right), 3.81(\mathrm{~s}, 3 \mathrm{H}), 5.11\left(\mathrm{~d},{ }^{3} J=3.4 \mathrm{~Hz}, 1 \mathrm{H}\right), 6.88$ $\left(\mathrm{d},{ }^{3} J=8.7 \mathrm{~Hz}, 2 \mathrm{H}\right), 7.23\left(\mathrm{~d},{ }^{3} J=8.7 \mathrm{~Hz}, 2 \mathrm{H}\right)$.

${ }^{13} \mathbf{C}-\mathbf{N M R}\left(\mathrm{CDCl}_{3}, 90 \mathrm{MHz}, 297 \mathrm{~K}\right): \delta 6.8$ (q), 28.4 (q), 33.5 (s), 50.2 (d), 55.4 (q), 73.0 (d), $113.6(\mathrm{~d}), 126.6(\mathrm{~d}), 138.4(\mathrm{~s}), 158.4(\mathrm{~s})$.

Spectroscopic Data of 2,3,3-Trimethyl-1-para-tolyl-butan-1-ol (4)

${ }^{1}$ H-NMR $\left(\mathrm{CDCl}_{3}, 360 \mathrm{MHz}, 297 \mathrm{~K}\right): \delta 0.91(\mathrm{~s}, 9 \mathrm{H}), 1.10\left(\mathrm{~d},{ }^{3} J=6.3 \mathrm{~Hz}, 3 \mathrm{H}\right), 2.34(\mathrm{~s}, 3 \mathrm{H})$, $3.12\left(\mathrm{q},{ }^{3} J=6.3 \mathrm{~Hz}, 1 \mathrm{H}\right), 4.36\left(\mathrm{~d},{ }^{2} J=11.7 \mathrm{~Hz}, 1 \mathrm{H}\right), 4.58\left(\mathrm{~d},{ }^{2} J=11.7 \mathrm{~Hz}, 1 \mathrm{H}\right), 7.14\left(\mathrm{~d},{ }^{3} J=\right.$ $8.1 \mathrm{~Hz}, 2 \mathrm{H}), 7.25\left(\mathrm{~d},{ }^{3} \mathrm{~J}=8.1 \mathrm{~Hz}, 2 \mathrm{H}\right)$.

${ }^{13} \mathbf{C}-\mathbf{N M R}\left(\mathrm{CDCl}_{3}, 90.6 \mathrm{MHz}, 297 \mathrm{~K}\right): \delta 14.0$ (q), 21.3 (q), 26.2 (q), 35.3 (s), 71.5 (t), 82.8 (d), 127.7 (d), 128.9 (d), 136.5 (s), 136.9 (s). 


\section{3. ${ }^{13} \mathrm{C}-\mathrm{NMR}$ spectra}

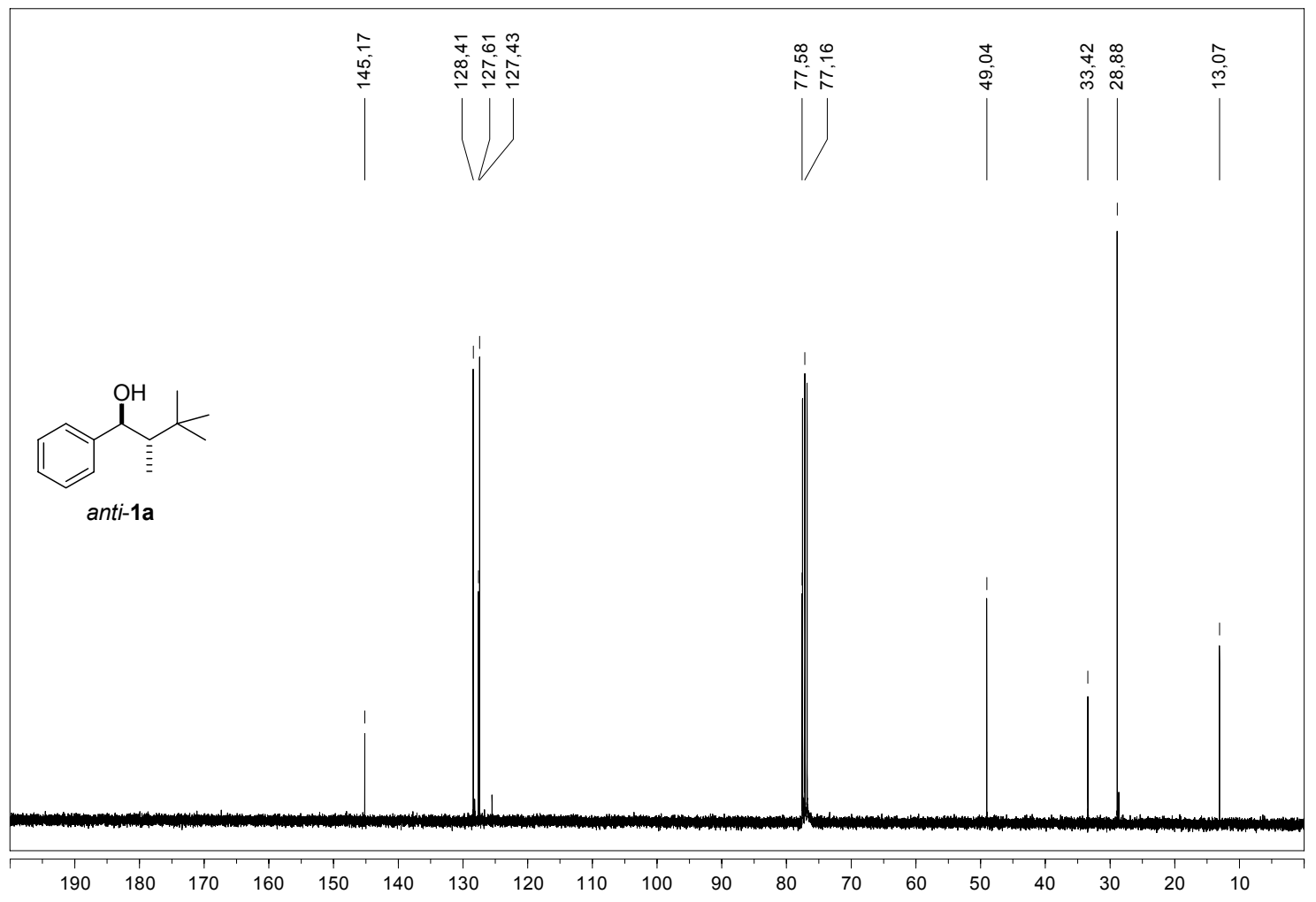
ppm (f1)

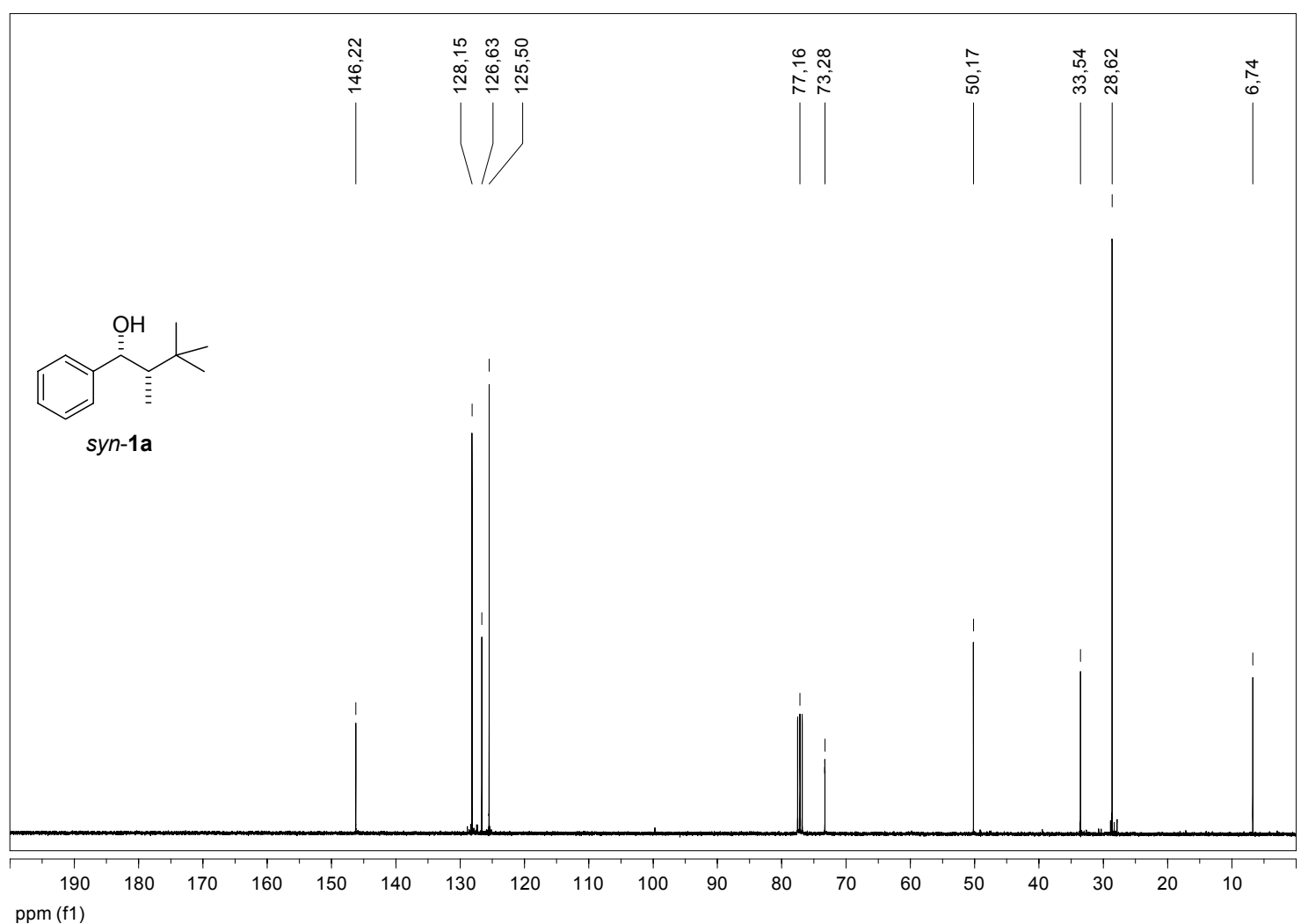



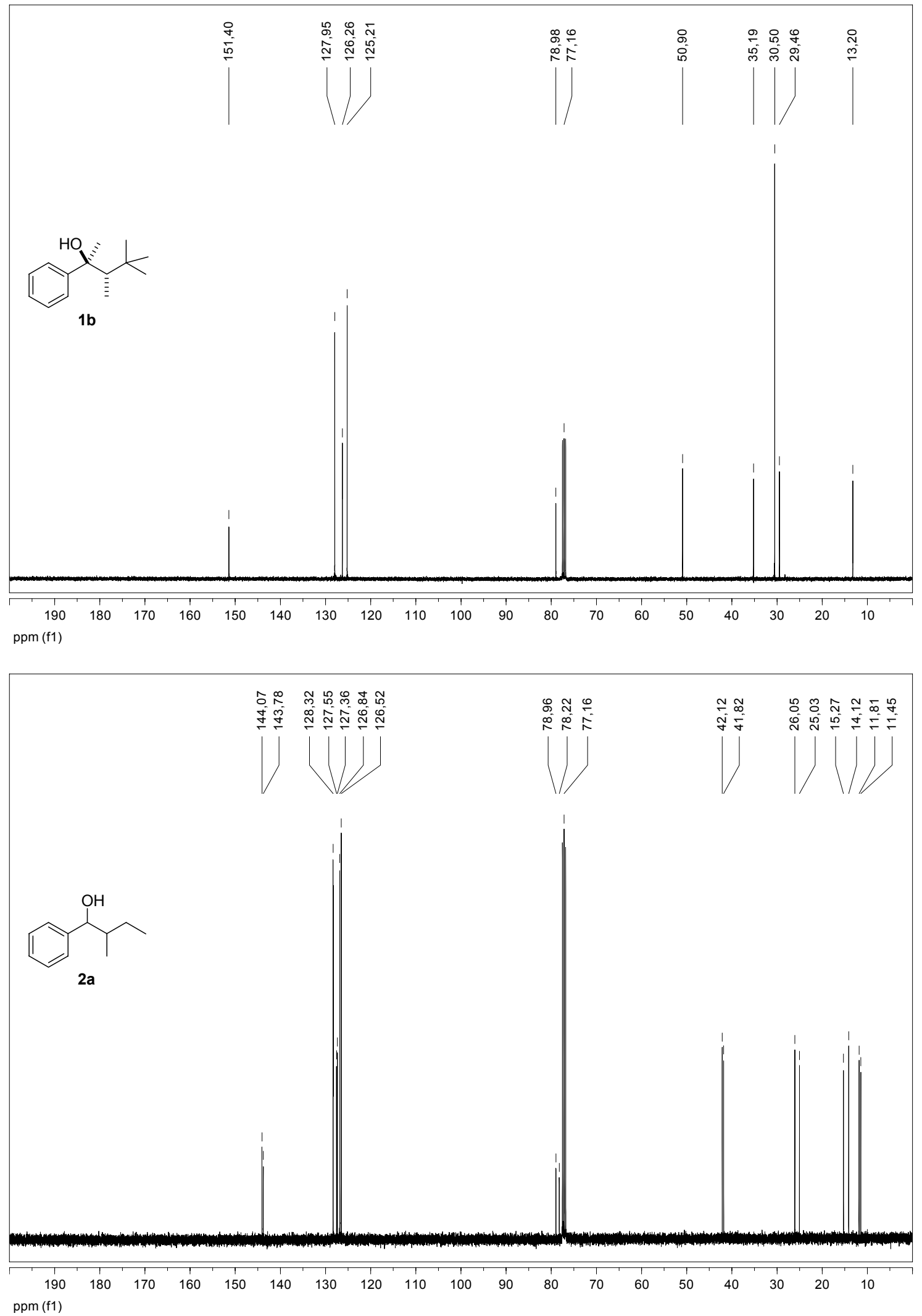

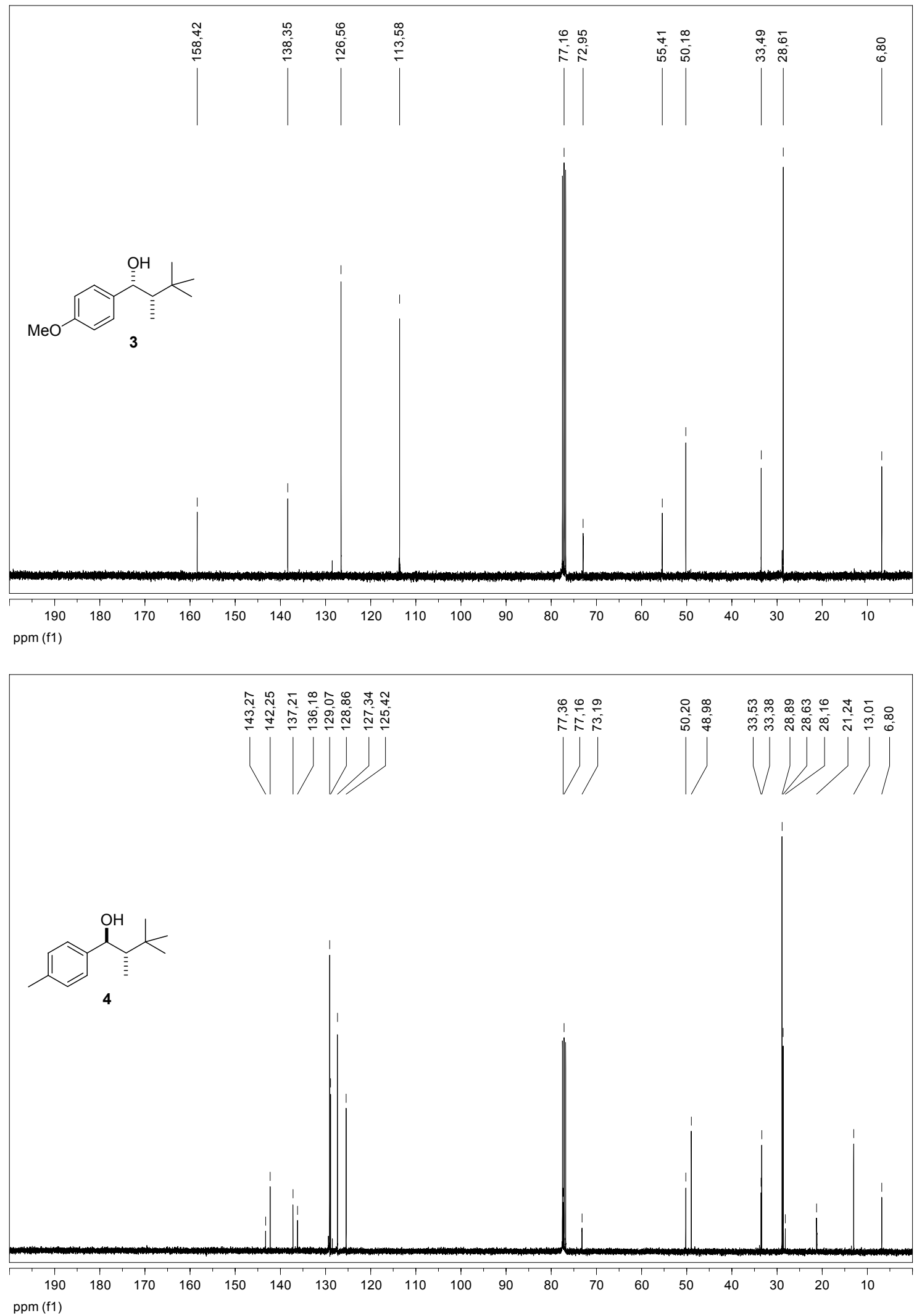

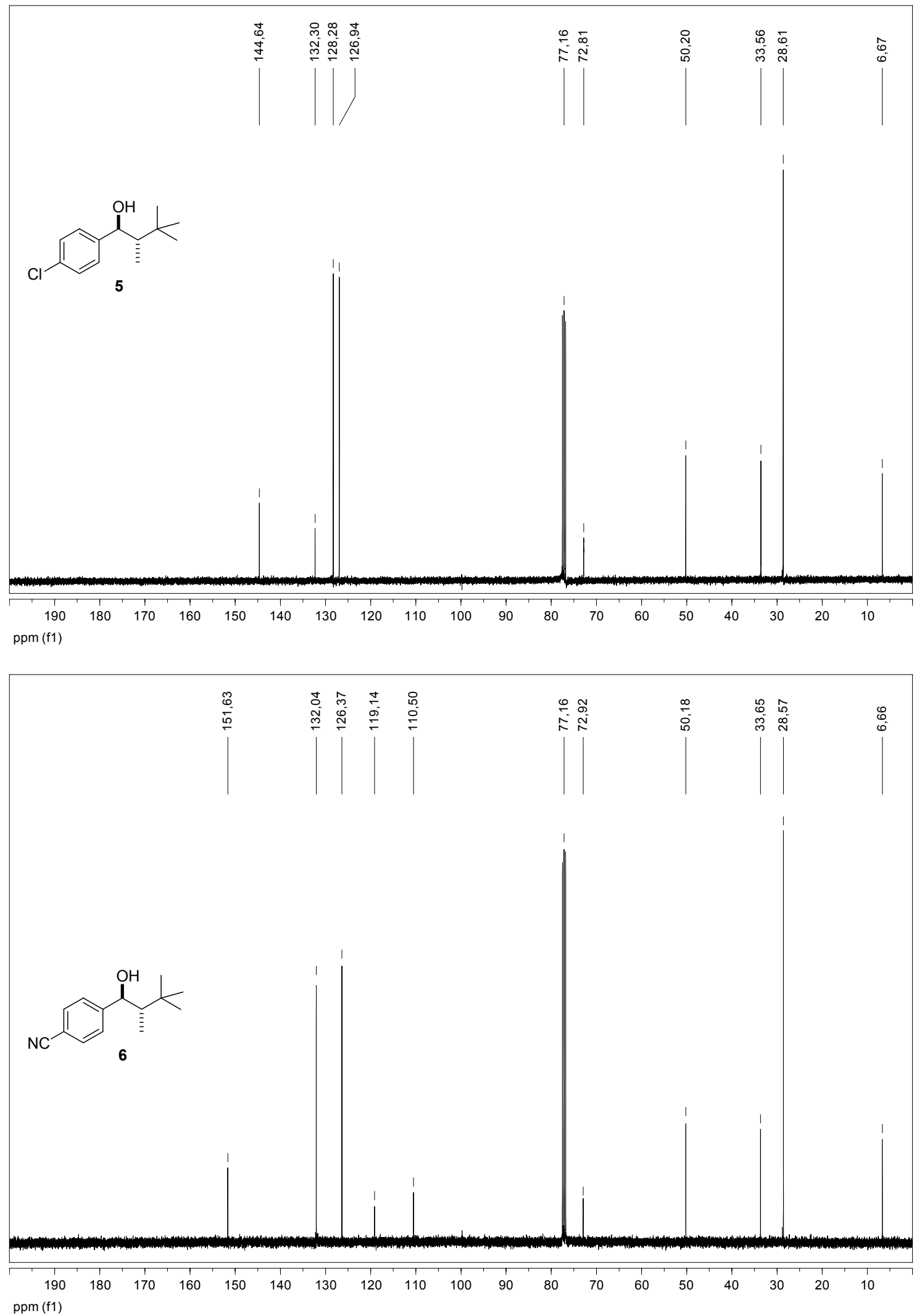

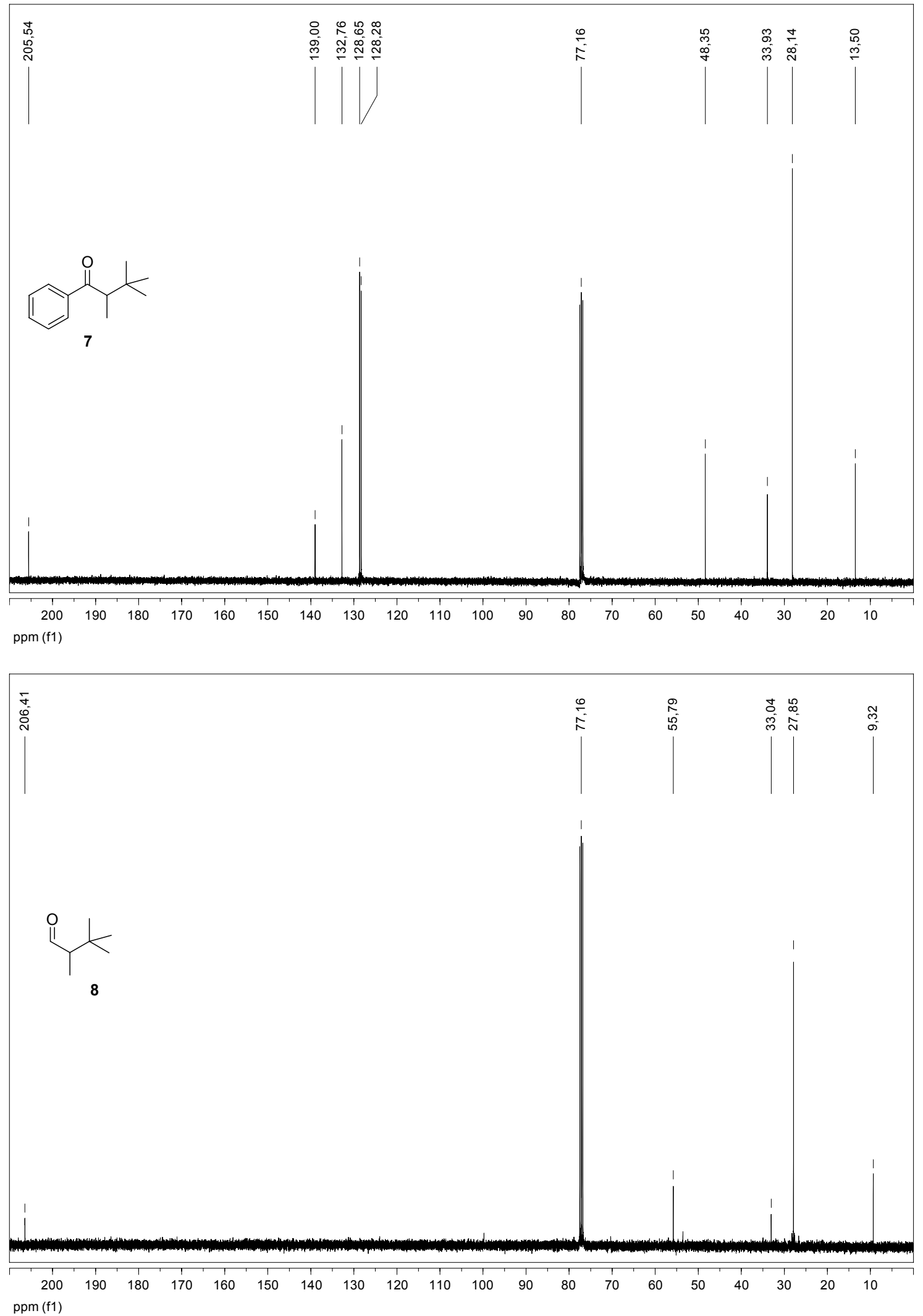

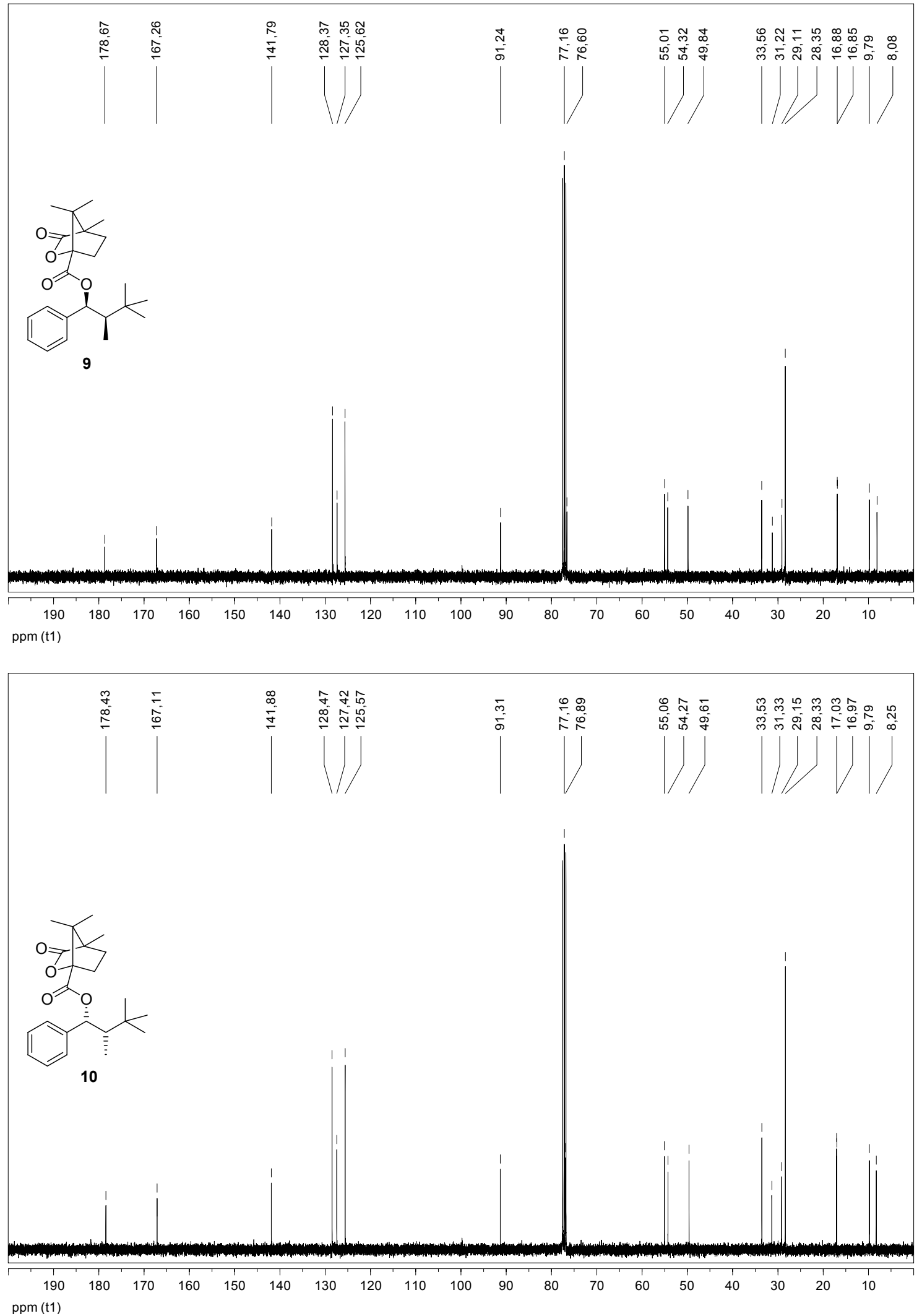

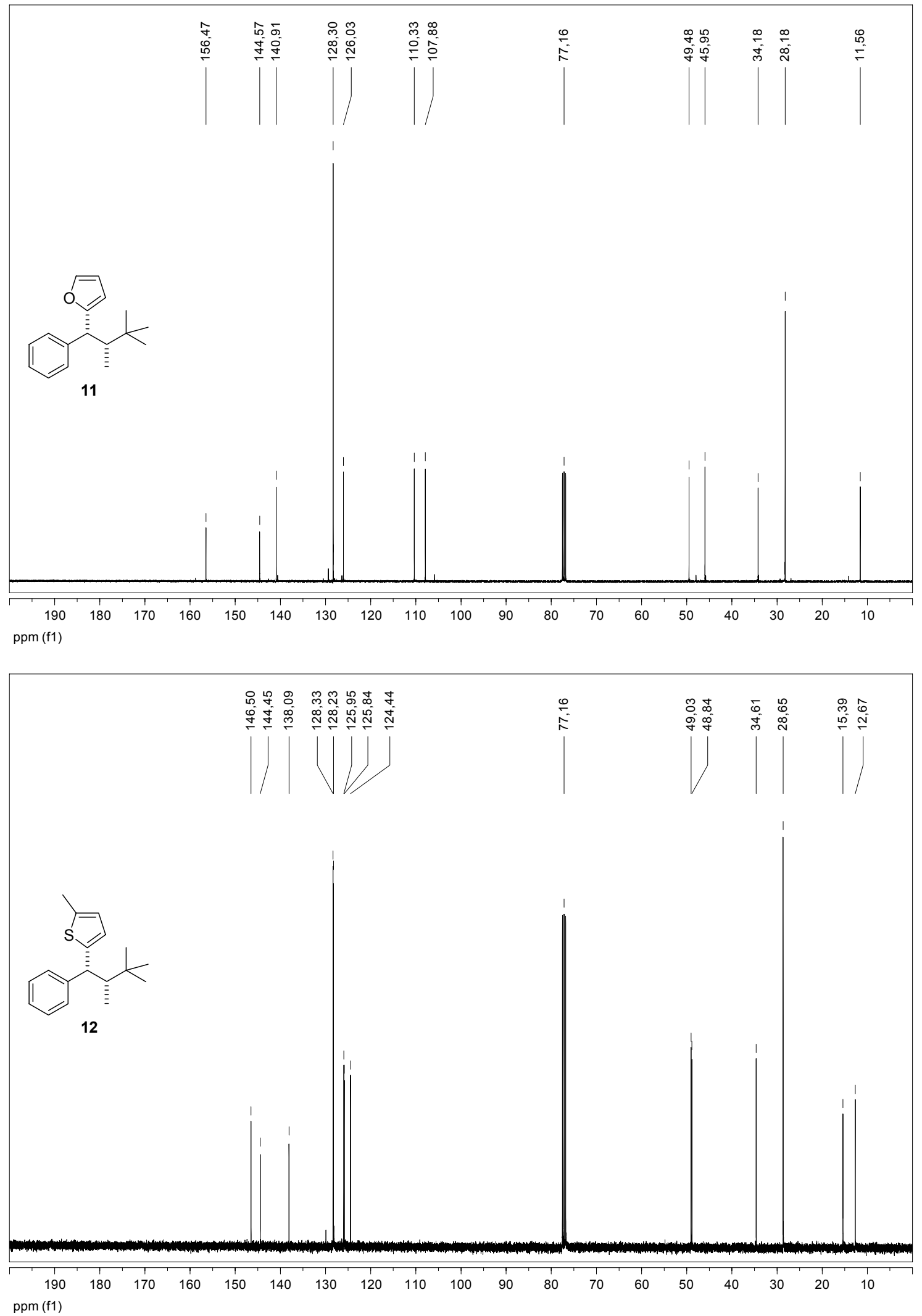


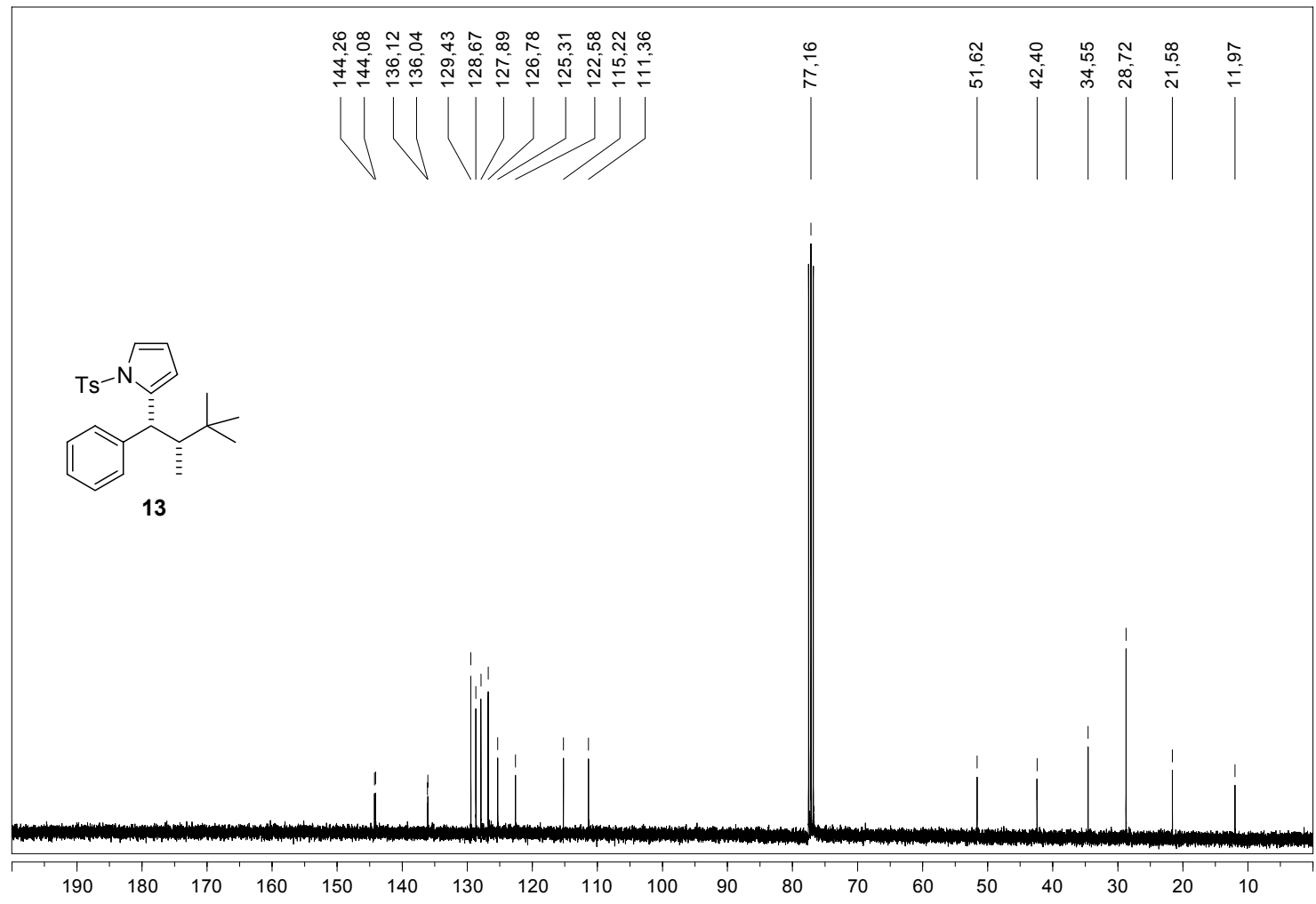
ppm (f1)

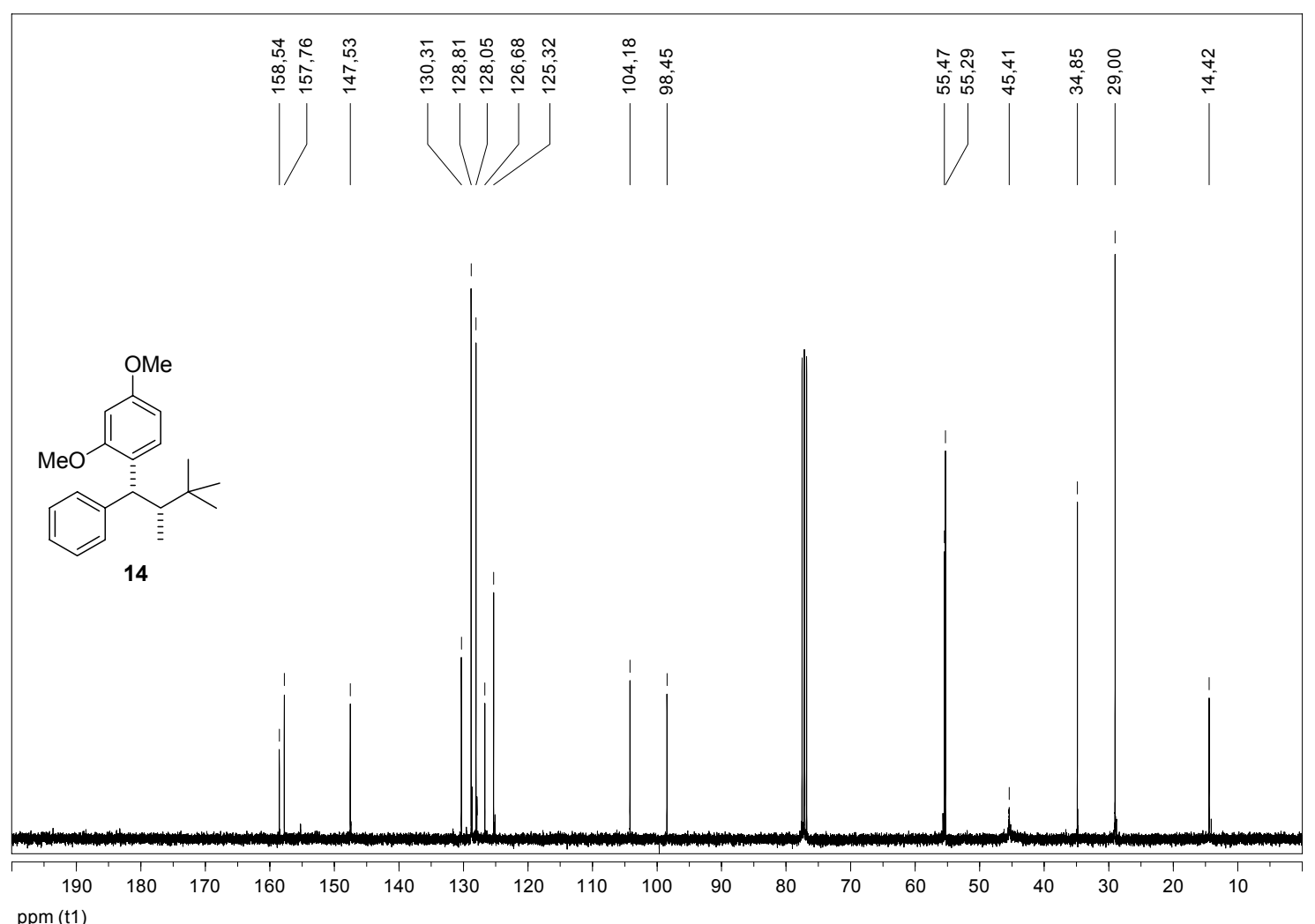




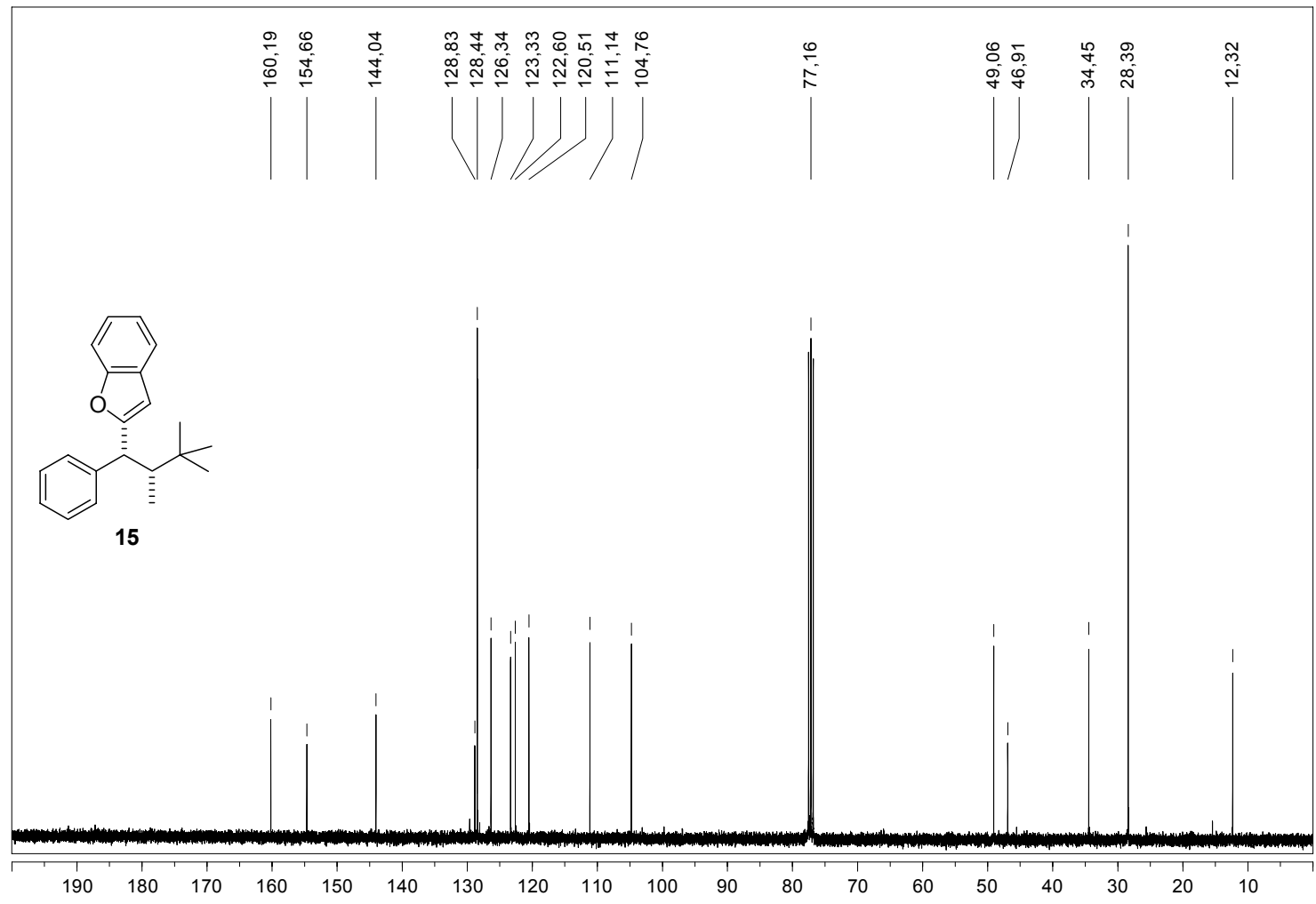

ppm (f1)

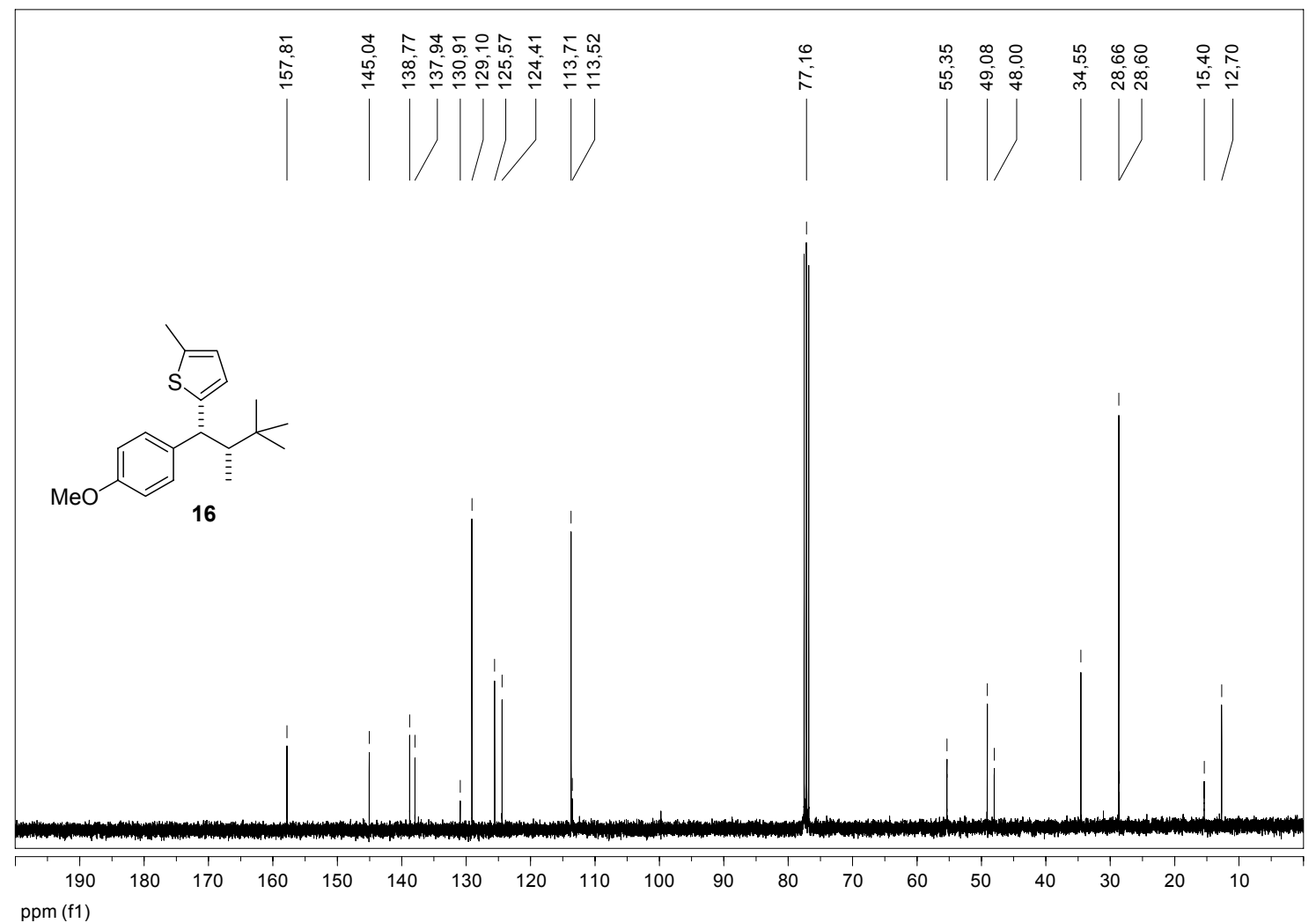



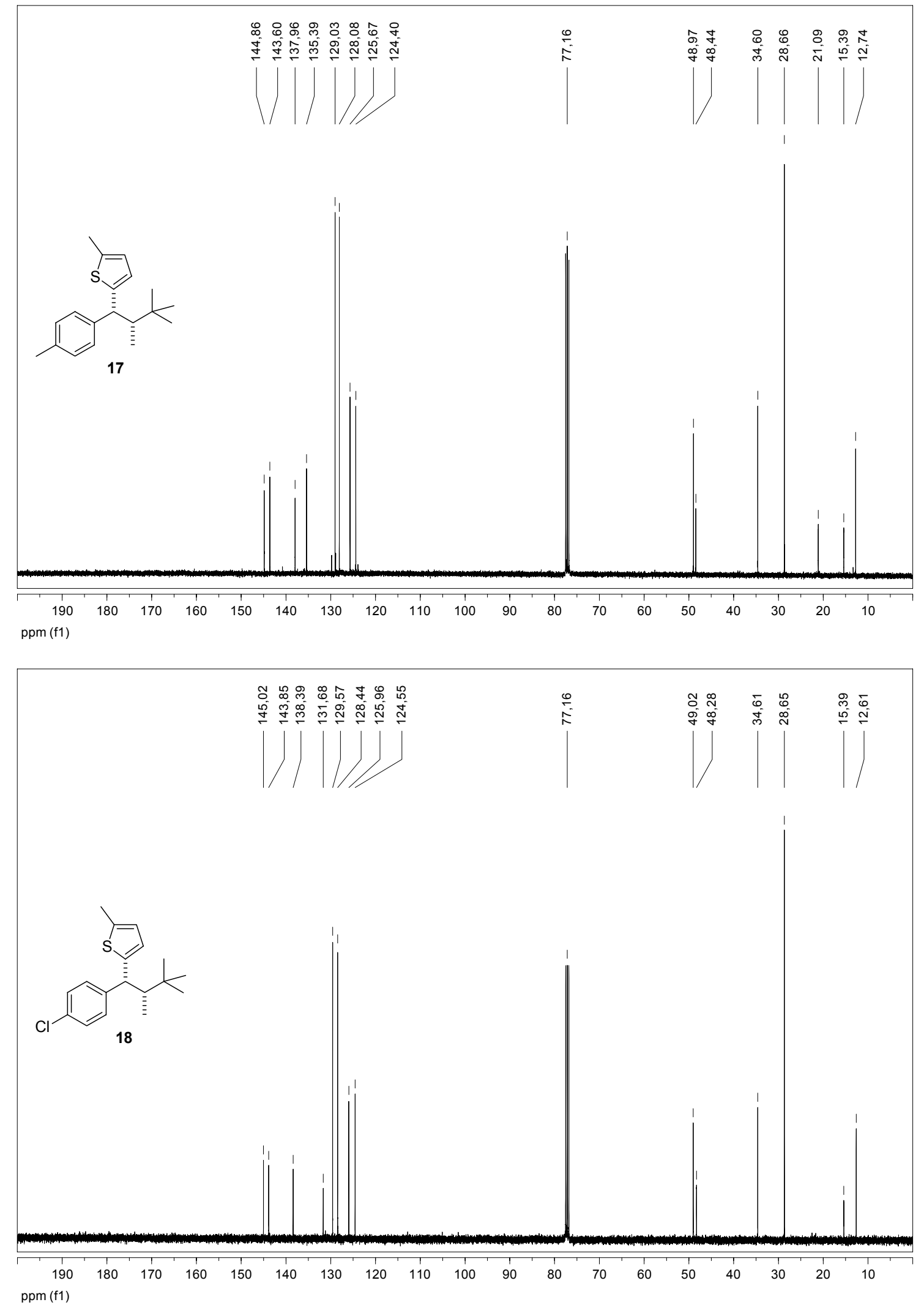


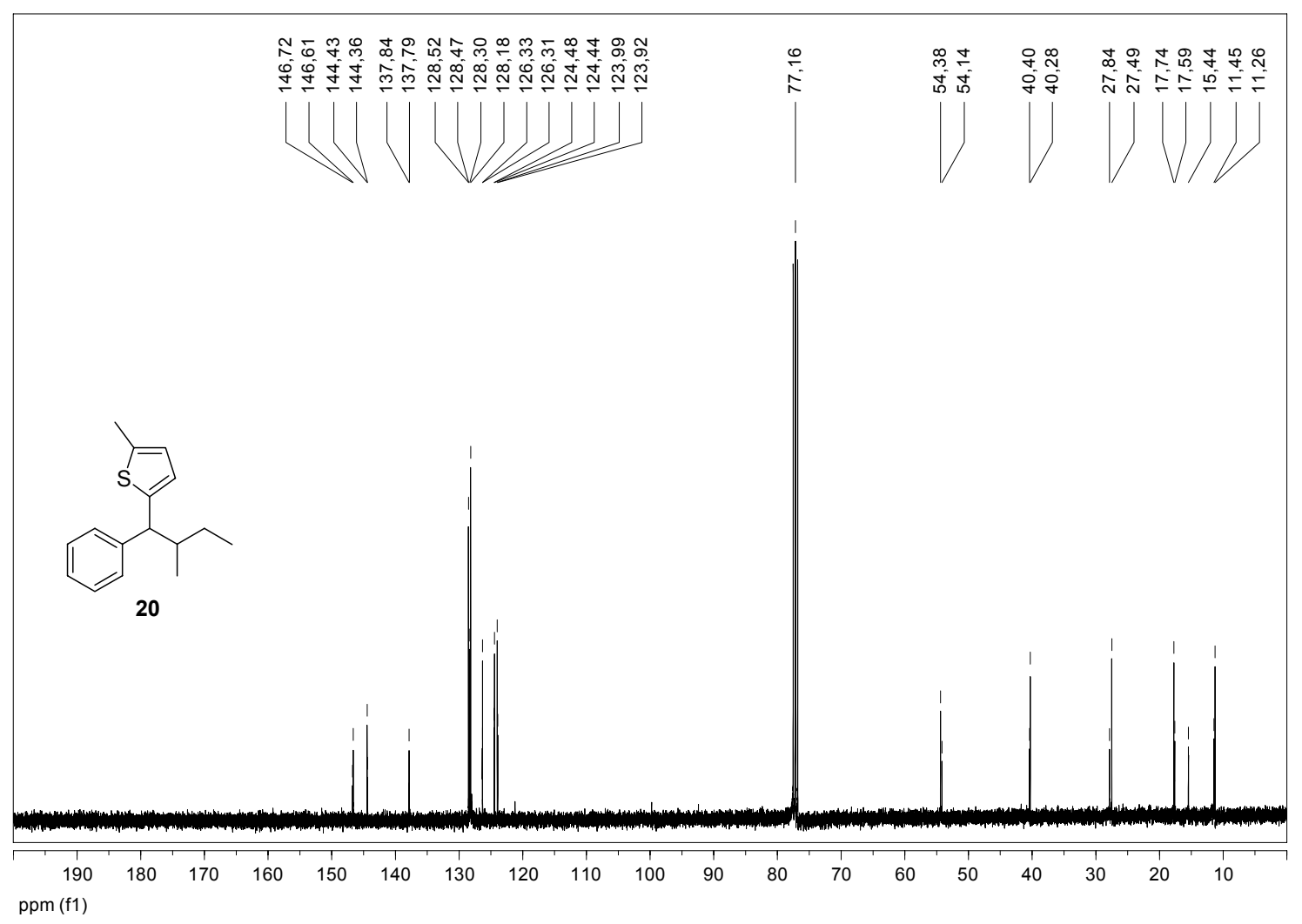




\section{NMR Studies}

${ }^{13}$ C NMR spectrum of cation 21

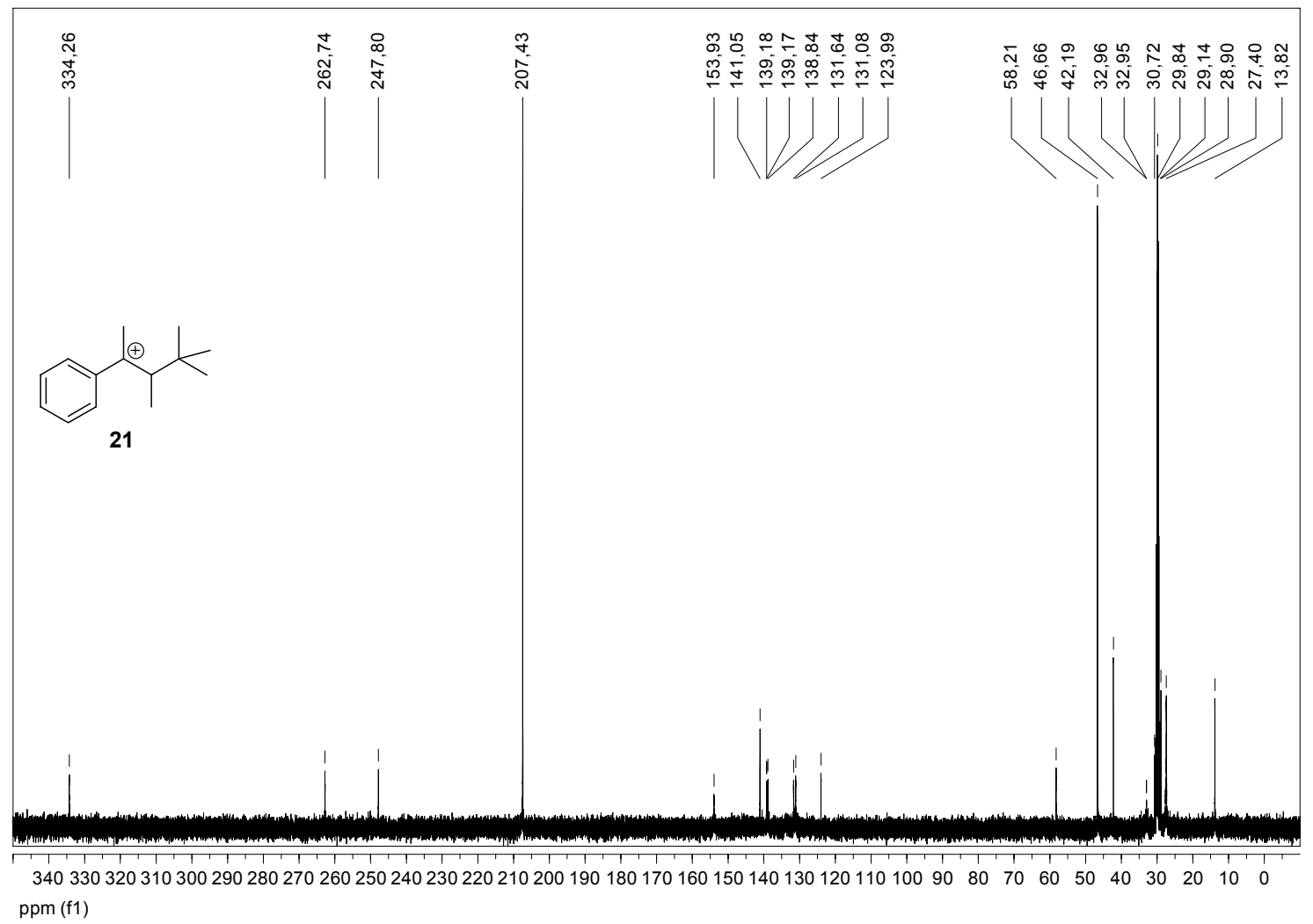




\section{${ }^{13} C$ NMR spectrum of cation 24}

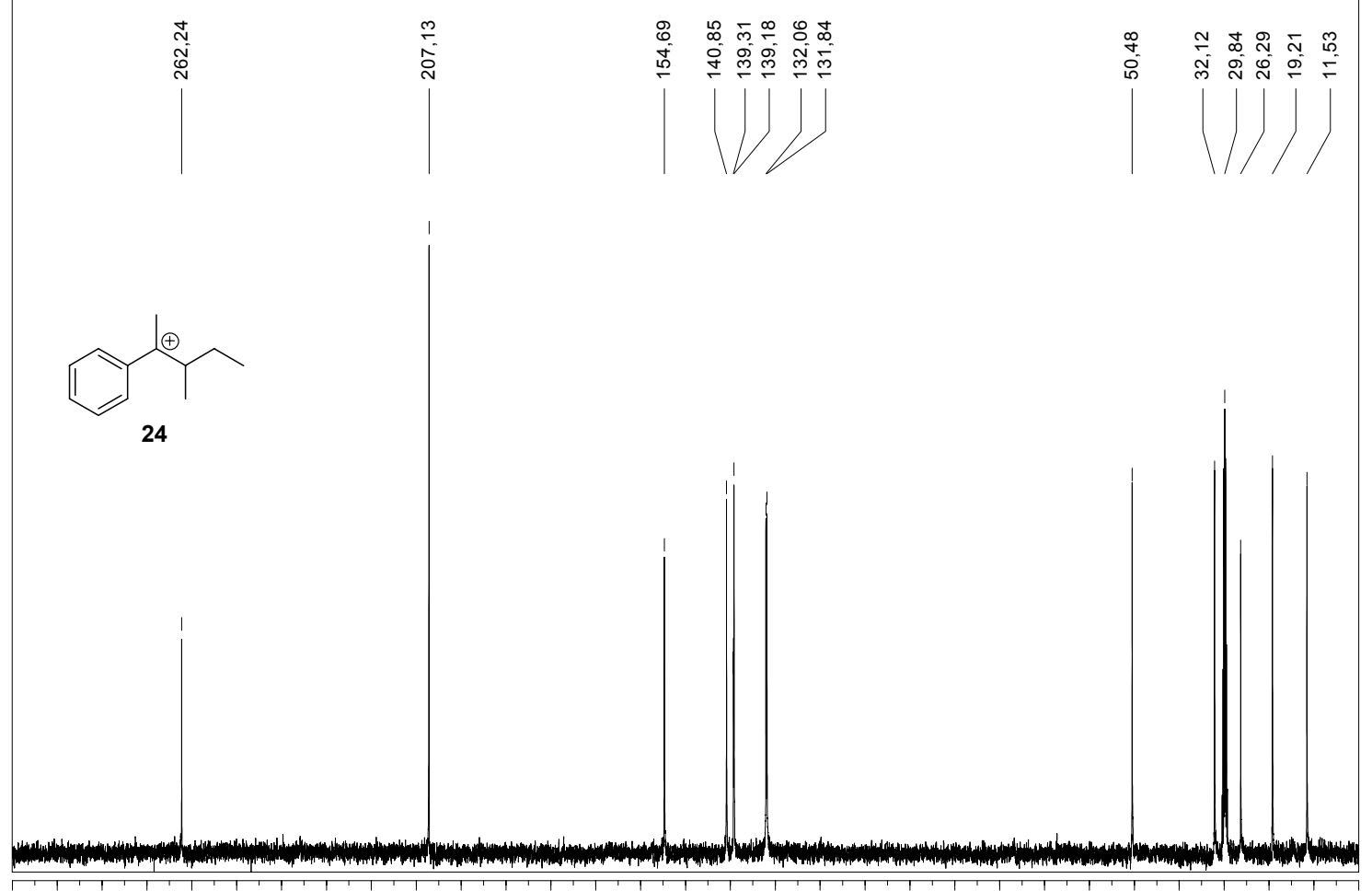

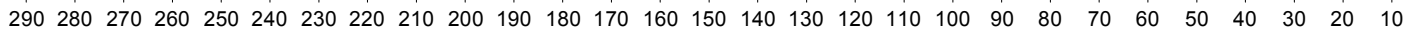
ppm (t1)

${ }^{1} H$ NMR spectrum of cation 24

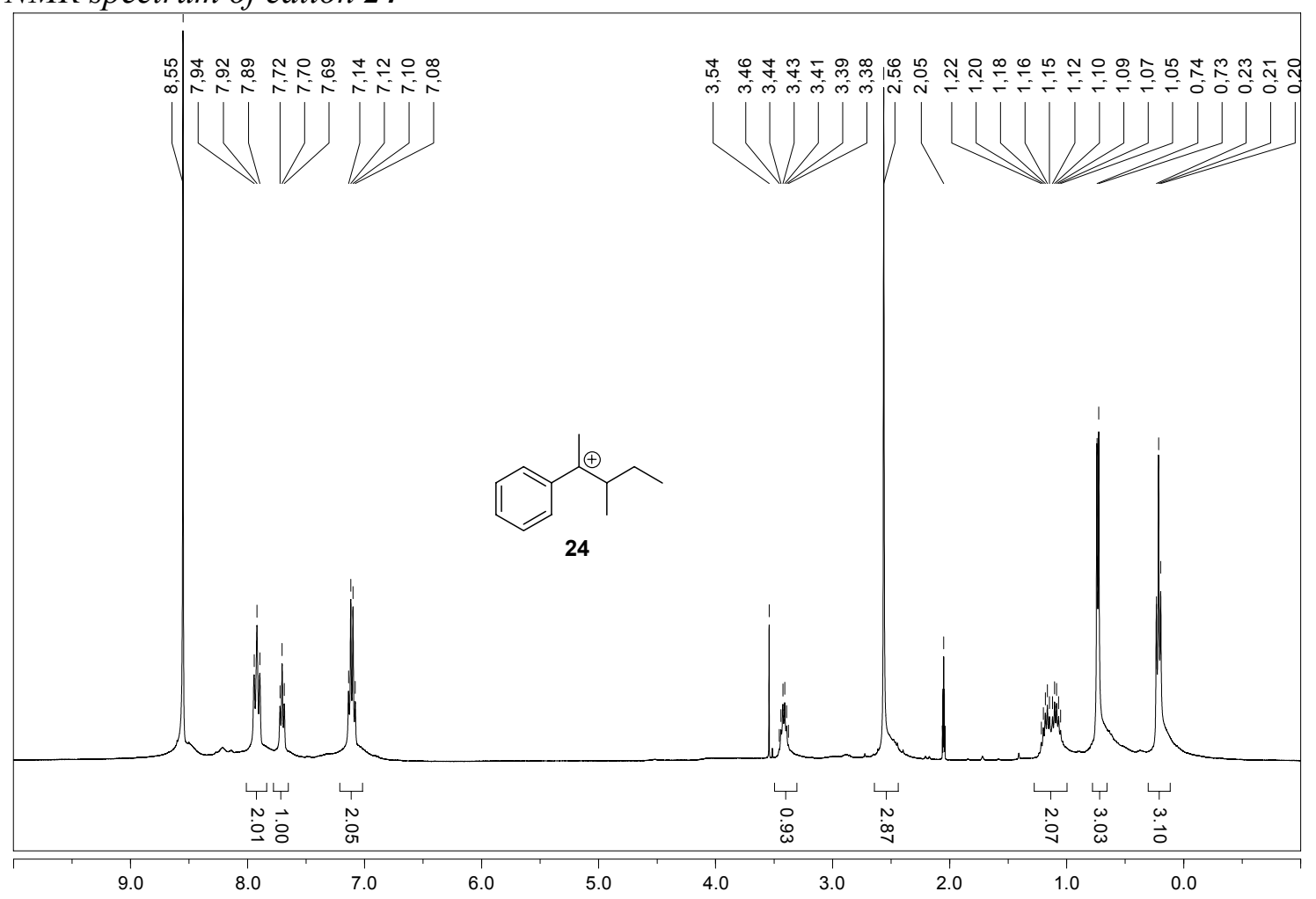

$\operatorname{ppm}(\mathrm{f} 1)$ 
1D NOESY spectrum of cation 24

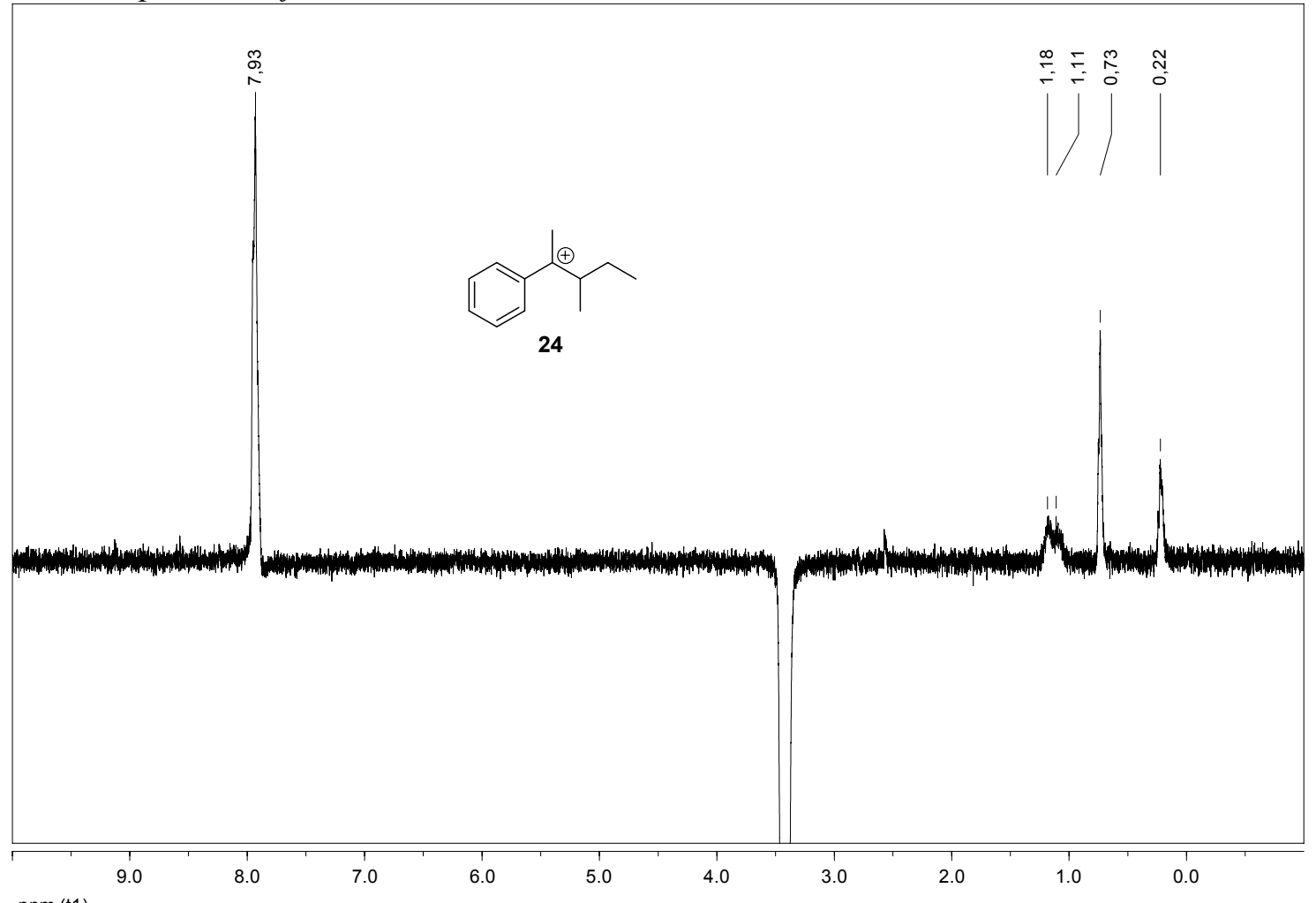

ppm (t1) 